\title{
The Algebra of Projective Spheres on Plane, Sphere and Hemisphere
}

\author{
István Lénárt \\ Eötvös Loránd University, Budapest, Hungary \\ Email: lenarti@caesar.elte.hu, h12572len@ella.hu
}

How to cite this paper: Lénárt, I. (2020) The Algebra of Projective Spheres on Plane, Sphere and Hemisphere. Journal of Applied Mathematics and Physics, 8, 2286-2333. https://doi.org/10.4236/jamp.2020.810171

Received: July 17, 2020

Accepted: October 27, 2020

Published: October 30, 2020

Copyright $\odot 2020$ by author(s) and Scientific Research Publishing Inc. This work is licensed under the Creative Commons Attribution International License (CC BY 4.0).

http://creativecommons.org/licenses/by/4.0/

\begin{abstract}
Numerous authors studied polarities in incidence structures or algebrization of projective geometry [1] [2]. The purpose of the present work is to establish an algebraic system based on elementary concepts of spherical geometry, extended to hyperbolic and plane geometry. The guiding principle is: "The point and the straight line are one and the same". Points and straight lines are not treated as dual elements in two separate sets, but identical elements within a single set endowed with a binary operation and appropriate axioms. It consists of three sections. In Section 1 I build an algebraic system based on spherical constructions with two axioms: $a b=b a$ and $(a b)(a c)=a$, providing finite and infinite models and proving classical theorems that are adapted to the new system. In Section Two I arrange hyperbolic points and straight lines into a model of a projective sphere, show the connection between the spherical Napier pentagram and the hyperbolic Napier pentagon, and describe new synthetic and trigonometric findings between spherical and hyperbolic geometry. In Section Three I create another model of a projective sphere in the Cartesian coordinate system of the plane, and give methods and techniques for using the model in the theory of functions.
\end{abstract}

\section{Keywords}

Projective Spheres, Binary Operation, Incidence Relation, Pappos, Desargues, Hesse-Chasles Configurations, Napier Pentagram and Hyperbolic Napier Pentagon, Polarity in Cartesian Coordinates, Differentiable Functions

\section{Introduction}

Since the beginning of my research in 1969 I have always tried to combine abstract theory with direct experimentation on real spheres in order to construct geometric figures and configurations. When studying the theoretical background, I was surprised at the stark contrast between the simple and elegant handling of 
plane geometry versus the much more complex way of discussing spherical geometry and trigonometry.

Eventually I came to the thought that the reason might be the Euclidean point of view in the non-Euclidean world of geometry. My conclusion was that from the very first steps spherical and hyperbolic geometry should be viewed from their own perspective, not through the Euclidean glasses.

This initiative has led me ever since. During the years, I have found a long list of predecessors in the topic from the ancient Greek scholar Menelaus of Alexandria to Leibniz [3] and other leading mathematicians of the last 400 years. The researchers from whom I received the strongest inspiration were Gerhard Hessenberg, Reinhold Baer, Vladimir Devidé and Ferenc Kárteszi in the $20^{\text {th }}$ century (See references in the text).

My problem was to construct a set of points and straight lines with a binary operation with few but efficient axioms. The purpose of this article is to generate interest in the central ideas of the theory and to briefly summarize the main results. More details can be found in [4].

\section{The Algebra of Projective Spheres}

\subsection{The Basic Idea}

Any point on the sphere determines the opposite point and the equator of the two points. In Riemannian geometry, any pair of opposite points constitute " $a$ point". Any two "points" determine one great circle through them, and any two great circles determine "a point" of intersection.

This construction does not lead to a binary operation, since we have two different sets of elements. Any two elements in one set determine an element in the other set, and conversely.

Consider the combination of a point, the opposite point and the equatorial line as the "basic element" of our geometry on the sphere. In other words, the basic element of our model is the natural polarity represented by a pair of pole points and the equatorial line on the sphere.

What is the advantage of the basic element so chosen? Let $x\left(X, X^{\prime}, x\right)$ denote a basic element with opposite points $X, X^{\prime}$, and equator $X$. Two basic elements $\boldsymbol{b}\left(B, B^{\prime}, b\right)$ and $\boldsymbol{c}\left(C, C^{\prime}, c\right)$ uniquely determine a third basic element $\boldsymbol{a}\left(A, A^{\prime}, a\right)$.

In this way, an algebraic structure with a binary operation is established in the set of spherical basic elements. The resulting element can geometrically be constructed from the factor elements in several ways. It is up to us to choose whatever representation is most convenient for us.

Figure 1 shows a basic element $\boldsymbol{a}\left(A, A^{\prime}, a\right)$ with pole points and equator; Figure 2, one great circle a passes through points $B, C$ or $B^{\prime}, C$ or $B^{\prime}, C$ or $B^{\prime}, C^{\prime}$; Figure 3, two great circles $b$ and $c$ intersect in two opposite points $A$, $A$; Figure 4 , two great circles $b$ and $c$ have one common perpendicular $a$; Figure 5, one perpendicular a can be dropped from point $B$ or $B^{\prime}$ to line $c$. 


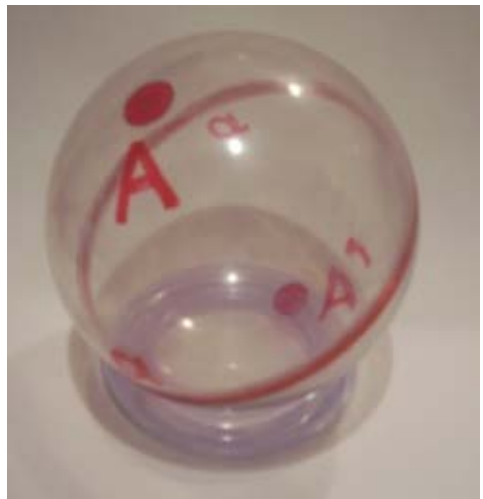

Figure 1. Polarity between pole points $A, A$ 'and great circle $a$.

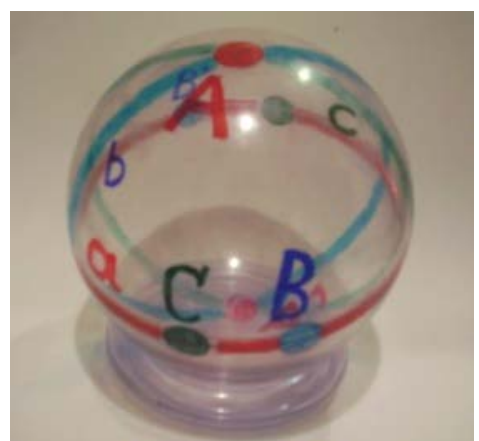

Figure 2. Great circle a passes through pole points $B, B^{\prime}$ and $C, C^{\prime}$.

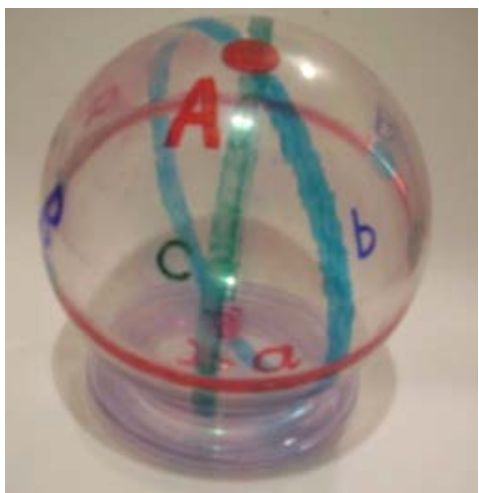

Figure 3. Great circles $b$, cintersect in pole points $A, A$ '.

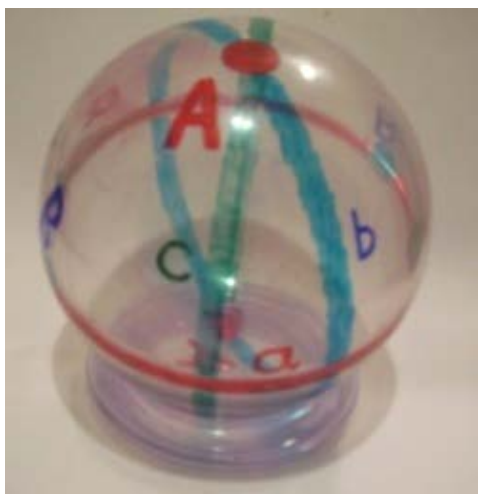

Figure 4. Great circles $b, c$, and common perpendicular $a$. 


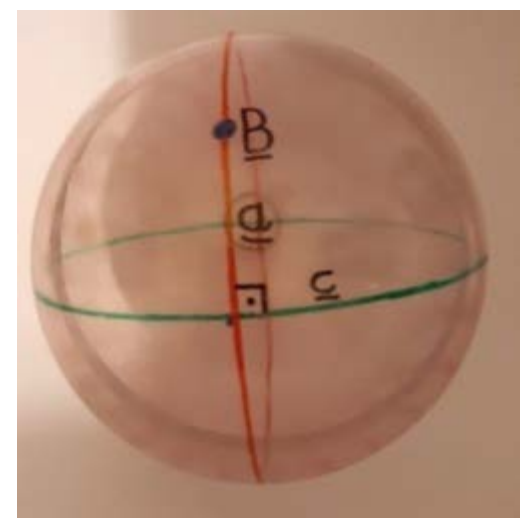

Figure 5. One perpendicular a from points $B, B^{\prime}$ to line $c$.

\subsection{Basic Algebraic Properties of the Operation}

Notation: Given elements $\boldsymbol{x}$ and $\boldsymbol{y}$, denote the operation by writing the two factors without any operation sign between them: $x \boldsymbol{y}=\mathbf{z}$.

At this stage the algebraic properties are proved by referring to the geometry of the sphere. Later on an axiomatic system is created to build the proofs on the axioms whenever possible.

Figure 6 shows that $\boldsymbol{x y}=\boldsymbol{y x}=\mathbf{z}$, commutative property is valid; Figure 7, $(\boldsymbol{a b})(\boldsymbol{a c})=\boldsymbol{a}$, a simple geometric property in algebraic form; Figure 8, if line $\boldsymbol{x}_{\boldsymbol{i}}$ coincides with line $a$, then $a x_{i}$ has infinitely many perpendiculars, so aa cannot be uniquely determined; Figure $9, \boldsymbol{a} \boldsymbol{b}=\boldsymbol{a c}$, but $\boldsymbol{b} \neq \boldsymbol{c}$, and $a, b, c$ are different, so cancellation does not work in this case.

Figure 10 shows that from $\boldsymbol{a} \boldsymbol{b}=\boldsymbol{a c}$ follows $\boldsymbol{a} \boldsymbol{b}=\boldsymbol{a} \boldsymbol{c}=\boldsymbol{b} \boldsymbol{c}$, the roles of the three elements are symmetric in this case; Figure 11, $\boldsymbol{a} \boldsymbol{b}=\boldsymbol{c d}$ gives

$\boldsymbol{a} \boldsymbol{b}=\boldsymbol{c d}=\boldsymbol{a d}=\boldsymbol{b} \boldsymbol{c}=\boldsymbol{a} \boldsymbol{c}=\boldsymbol{b} \boldsymbol{d}$; Figure 12, general unit element does not exist in the set (a straight line cannot be perpendicular to itself); Figure 13, from $\boldsymbol{a} \boldsymbol{b}=\boldsymbol{c}$ does not follow $\boldsymbol{b c}=\boldsymbol{a}$ (in contrast with Steiner triplets), but $\boldsymbol{a} \boldsymbol{b}=\boldsymbol{c}$ and $\boldsymbol{b} \boldsymbol{c}=\boldsymbol{a}$ give $\boldsymbol{c a}=\boldsymbol{b}$.

Figure 14 shows that $a, a b,(a b) a$ make a triangle with three right angles, and the same is valid for $b, a b,(a b) b$; Figure 15, $[(\boldsymbol{a} b) \boldsymbol{c}] \boldsymbol{a}=[(\boldsymbol{a b}) \boldsymbol{c}] \boldsymbol{b}=\boldsymbol{a} \boldsymbol{b}$, we can bring out $a b$ from the parentheses if mediator $c$ exists, but $(a b) a$ or $(a b) b$ cannot be simplified in the general case; Figure 16, $\boldsymbol{a}$ and $\boldsymbol{b}$ are in incidence relation if poles of $\boldsymbol{a}$ on equator of $\boldsymbol{b}$, or poles of $\boldsymbol{b}$ on equator of $\boldsymbol{a}$ or equators of $\boldsymbol{a}$ and $\boldsymbol{b}$ are perpendicular, or pole of $\boldsymbol{a}$ at $90^{\circ}$ distance from pole of $\boldsymbol{b}$; Figure 17, inverse operation: Given $\boldsymbol{a}$ and $\boldsymbol{e}$ in $\boldsymbol{a x}=\boldsymbol{e}$, what is $\boldsymbol{x}$ ? If $\boldsymbol{a}$ and $\boldsymbol{e}$ are incident, there are infinitely many solutions for $\boldsymbol{x}$, but if $\boldsymbol{a}$ and $\boldsymbol{e}$ are not incident, no solution exists for $\boldsymbol{x}$.

Figure 18 shows the symmetry of the incidence relation: If equation $\boldsymbol{a} \boldsymbol{x}=\boldsymbol{b}$ has at least one solution for $\boldsymbol{x}$, equation $\boldsymbol{b y}=\boldsymbol{a}$ has at least one solution for $\boldsymbol{y}$, for example, $\boldsymbol{a} \boldsymbol{x}=\boldsymbol{b}$, choose $\boldsymbol{y}=\boldsymbol{a} \boldsymbol{b}$, then $\boldsymbol{b}(\boldsymbol{a} \boldsymbol{b})=(\boldsymbol{a x})(\boldsymbol{a b})=\boldsymbol{a}$; Figure 19, the associative element $(a b) c$ is an altitude of the triangle with sides $a, b, c$, since it is perpendicular to side $c$ through vertex $a b$, while the other two altitudes are 
(bc) $a$ and ( $c a) b$; Figure 20, the altitudes of a triangle do not coincide in the general case, so the associative property $(\boldsymbol{a b}) \boldsymbol{c}=\boldsymbol{a}(\boldsymbol{b c})=\boldsymbol{b}(\boldsymbol{c a})$ does not hold in the set; Figure 21, the product of any two of the three altitudes in a non-degenerate spherical triangle is the same:

$[(\boldsymbol{a b}) \boldsymbol{c}][(\boldsymbol{b c}) \boldsymbol{a}]=[(\boldsymbol{b c}) \boldsymbol{a}][(\boldsymbol{c a}) \boldsymbol{b}]=[(\boldsymbol{c a}) \boldsymbol{b}][(\boldsymbol{a} \boldsymbol{b}) \boldsymbol{c}]$, the orthocentre of the spherical triangle.

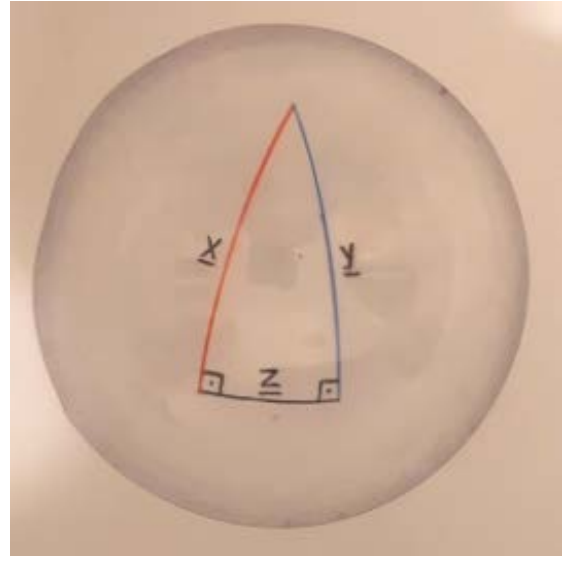

Figure 6. $x y=y x=z$.

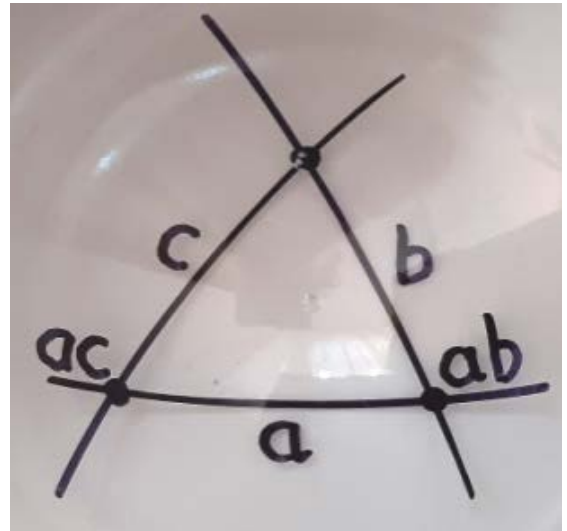

Figure 7. $(a b)(a c)=a$.

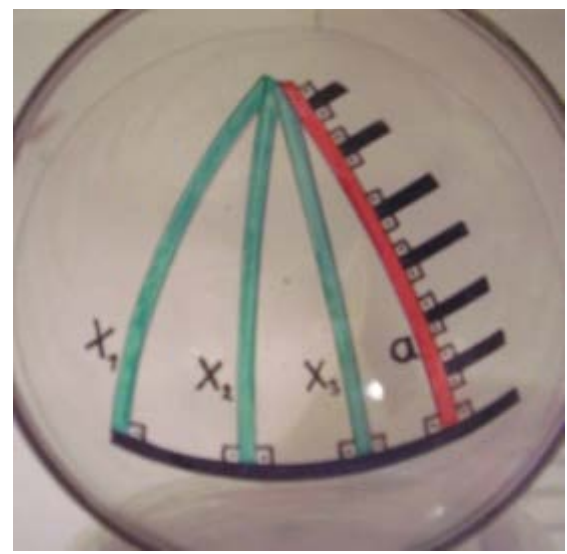

Figure 8. a undefined. 


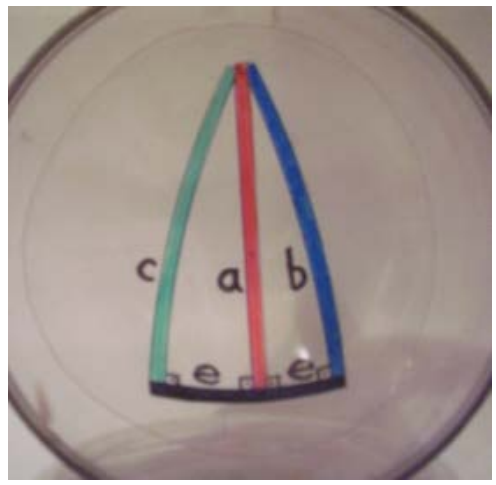

Figure 9. $a b=a c \nRightarrow b=c$.

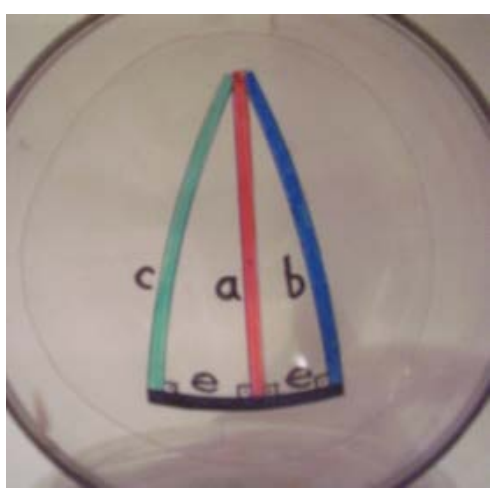

Figure 10, $a b=a c \Rightarrow a b=a c=b c$.

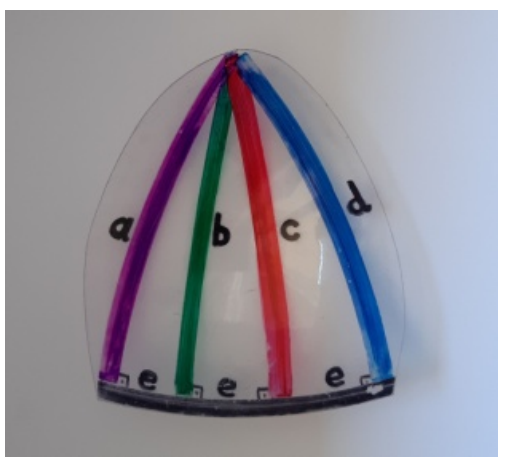

Figure 11. $a b=c d \Rightarrow a b=c d=a d=b c=a c=b d$

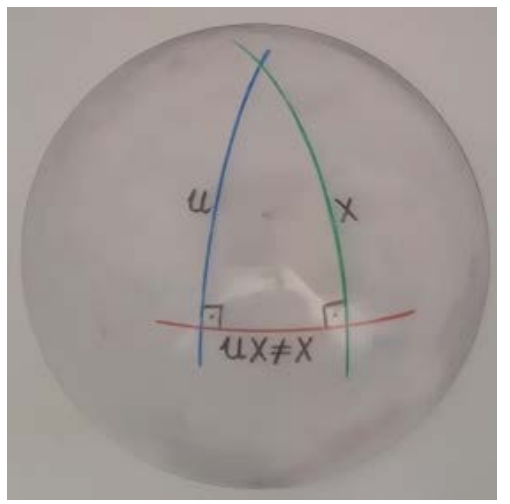

Figure 12. $u x \neq x$. 


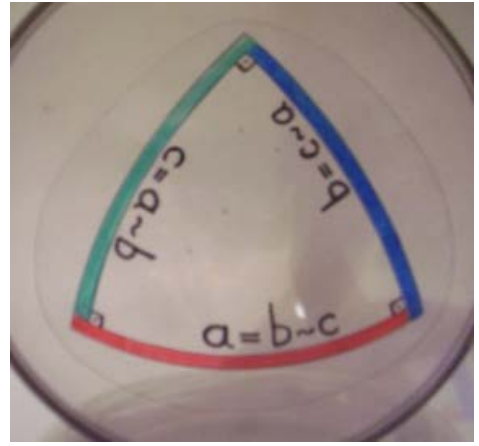

Figure 13. $a b=c, b c=a \Rightarrow c a=b$.

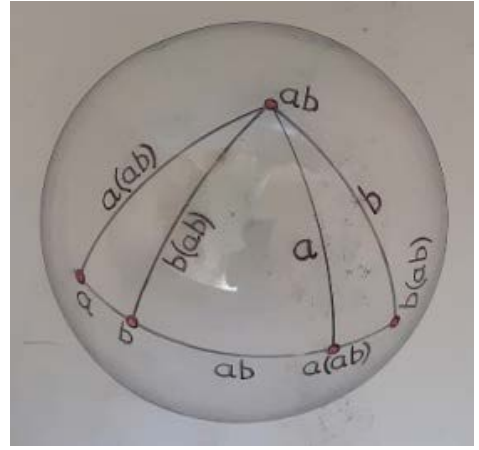

Figure 14. $a, a b,(a b) a$.

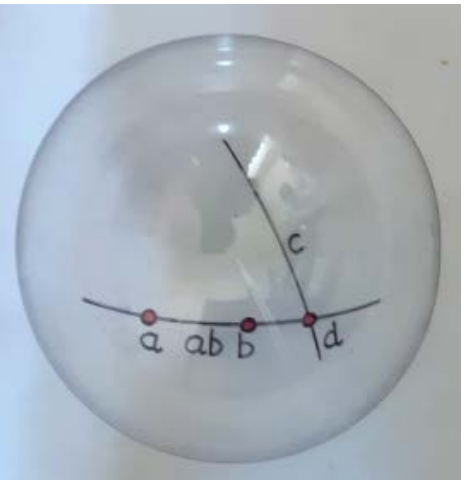

Figure 15. $[(a b) c] a=[(a b) c] b=a b$.

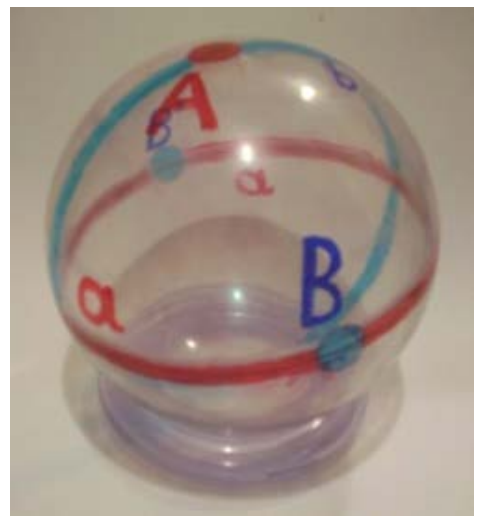

Figure 16. Incidence. 


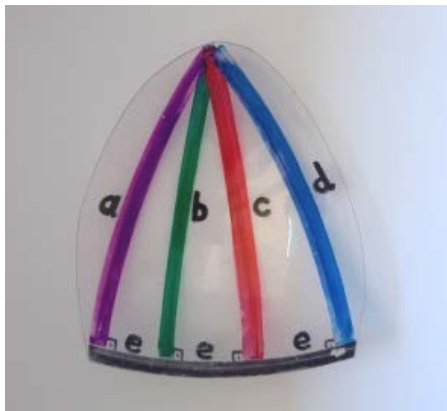

Figure 17. Inverse.

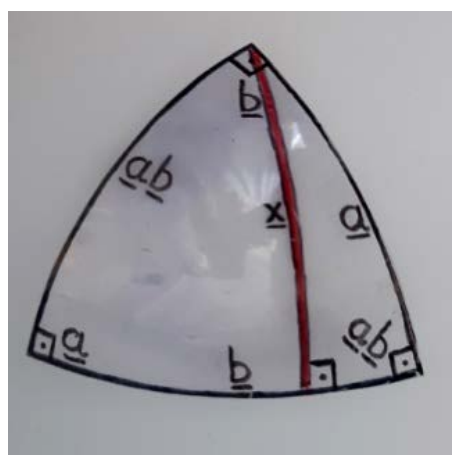

Figure 18. $a x=b \Rightarrow b y=a$.

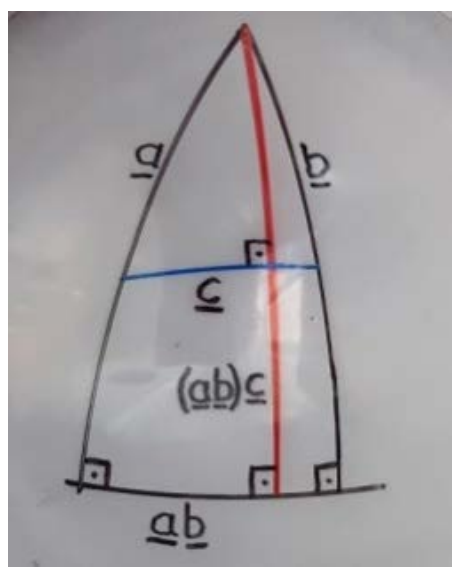

Figure 19. $(a b) c$ altitude of $a, b, c$.

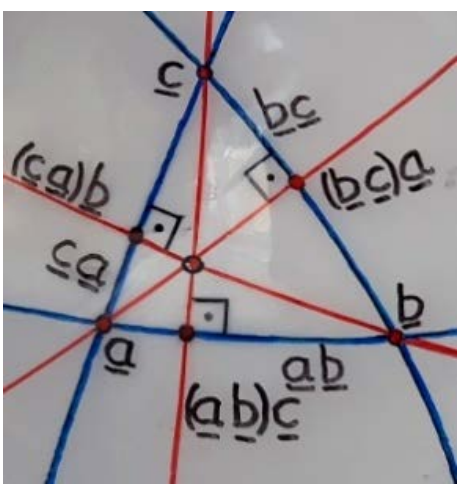

Figure 20. $(a b) c \neq(b c) a$. 


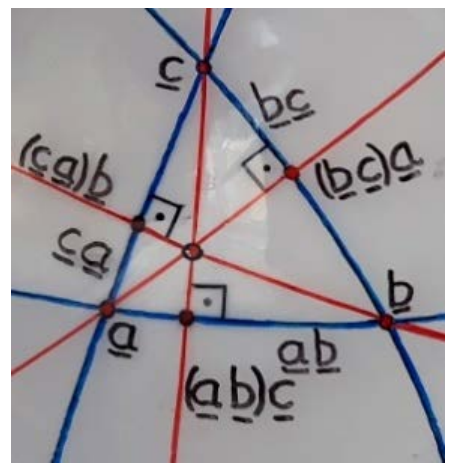

Figure 21. Orthocentre.

\subsection{Choose Axioms from the above Properties}

Consider a set of elements with a binary operation between different elements of the set:

$$
x y=z(x \neq y)
$$

Choose two axioms (Figure 6 and Figure 7):

1) $\boldsymbol{a} \boldsymbol{b}=\boldsymbol{b} \boldsymbol{a}$ (commutative);

2) $(\boldsymbol{a b})(\boldsymbol{a c})=\boldsymbol{a}($ main axiom $)$.

Call a set that satisfies these axioms a projective sphere.

Note that the product $\boldsymbol{x} \boldsymbol{x}$ is not disposed of in the axioms. The axioms only claim that $a b=b a$ if $a \neq b$; and $(a b)(a c)=a$ if $a \neq b, a \neq c, a b \neq a c$. The $\boldsymbol{x} \boldsymbol{x}$ case will be examined below.

\subsection{Binary Operation vs. Binary Relation}

The above definition of projective spheres is built on a binary operation.

Another option is to start with a binary relation based on the notion of incidence between points and lines [5] [6] [7].

Consider a set of elements with a binary relation $R$ defined between any two elements of the set, including the $\boldsymbol{x} \boldsymbol{R} \boldsymbol{x}$ case, for which the following two axioms hold:

1) $\boldsymbol{x} \boldsymbol{R} \boldsymbol{y}=\boldsymbol{y} \boldsymbol{R} \boldsymbol{x}$ (symmetric relation);

2) $\exists ! z \mid x \neq y \Rightarrow x \boldsymbol{R} z \boldsymbol{R} \boldsymbol{y}$.

(any two different elements $\boldsymbol{x} \neq \boldsymbol{y}$ determine one element $\boldsymbol{z}$ for which $\boldsymbol{z} \boldsymbol{R} \boldsymbol{X}$ and $z R y)$

The operation can be deduced from the relation or conversely. The present paper focuses on the operation. That is why the main axiom $(\boldsymbol{a b})(\boldsymbol{a c})=\boldsymbol{a}$ plays a key role, similar to the associative axiom $\boldsymbol{a}(\boldsymbol{b c})=(\boldsymbol{a} \boldsymbol{b}) \boldsymbol{c}$ in the theory of groups and fields. Nevertheless, the relation is also frequently used when it is more viable, mainly in Section Two and Section Three.

\subsection{Theorems Deduced from the Axioms}

Note that the theorems and proofs are based only on the algebra of projective spheres, without reference to geometric properties. 
Theorem 1 (Figure 8): aa cannot be defined for all elements of the set with at least two different elements.

At first sight, this property seems a trifle, no more than a remark. Actually, it is of fundamental importance for the whole system. In his theory that relates to the present one in several ways, Devidé ranked this property among the axioms [5]. I deduce it as a theorem from the two axioms.

Proof: I prove the following: If there is at least two different elements $a \neq b$ in the set, the expression $(a b)(a b)$ cannot be well defined for all elements of the set. The proof is short: $(a b)(a b)=a=(b a)(b a)=b$, which contradicts $a \neq b$. Q.E.D.

Consequence: This is a partial binary operation with aa undefined (Cf. division by zero among numbers). It cannot be extended into a non-partial operation that defines the product of any two elements, including identical elements.

Note that the proof does not preclude the option that aa is interpreted for a subset of the set.

Theorem 2 (Figure 10): From $a b=a c$ it follows that $a b=a c=b c$.

Proof: The assumption needs to be refined. If $b=c$, then $a b=a c$, but $b c$ cannot be performed because of Theorem 1 that was already proved.

From $a b=a c$ it follows that either $b=c$, or if $b \neq c$, then $a b=a c=b c$ (We need not specify $a \neq b, a \neq c$, because the existence of products $a b$ and $a c$ exclude $a=b$ and $a=c$ ).

If $a b=a c, b \neq c$, suppose by indirect proof that $a b \neq b c$; then $a c \neq b c$. We can multiply both sides of equation $a b=a c$ by $b c$, because the factors are different, and Theorem 1 cannot interfere. This gives $(a b)(b c)=(a c)(b c)$. By the axioms we get $(a b)(b c)=b=(a c)(b c)=c$ which contradicts to $b \neq c$. The only way out of the contradiction is the assumption that $a b=a c=b c$, because in this case Theorem 1 excludes the operations $(a b)(b c)$ and $(a c)(b c)$. Q.E.D.

Note that Theorem 1 plays a key role in the proof.

Theorem 3 (Figure 11): From $a b=c d$ follows $a b=c d=a d=b c=a c=b d$.

( $a, b, c, d$ are all different.)

Proof (similar to Theorem 2): If $a b=c d$, suppose by indirect proof that $a b \neq b d$; then $c d \neq b d$. We can multiply both sides of equation $a b=c d$ by $b d$, because the factors are different, and Theorem 1 cannot interfere. This gives $(a b)(b d)=(c d)(b d)$. By the axioms we get $(a b)(b d)=b=(c d)(b d)=d$ which contradicts $b \neq d$. The only escape from the contradiction is to assume that $a b=c d=b d$, for in this case Theorem 1 forbids to execute operations $(a b)(b d)$ and $(c d)(b d)$.

Same for any other equality in $a b=c d=a d=b c=a c=b d$. Q.E.D.

Theorem 4 (Figure 13): From $a b=c$ and $b c=a$ follows $c a=b$.

Proof (almost trivial): $c a=(a b)(b c)=b$. Q.E.D.

Theorem 5 (Figure 14): $a, a b,(a b) a$ make an octant, a triangle with three right angles. This means that the product of any two elements among $a, a b,(a b) a$ gives the third element. 
Proof: Denote $a=K, a b=L,(a b) a=M$.

$K L=a(a b)=M \quad$ (trivial),

$L M=(a b)[a(a b)]=a=K \quad$ (main axiom, $a$ repeats in factors $a b$ and $a(a b)$ )

$M K=[(a b) a] a=($ use $L M=(a b)[a(a b)]=a)=[(a b) a]\{(a b)[a(a b)]\}=a b=L$

(main axiom, $a b$ repeats in factors $(a b) a$ and $(a b)[a(a b])$

Similarly for $b, a b,(a b) b$. Q.E.D.

Theorem 6 (Figure 15): Simplify an expression: $[(a b) c] a=[(a b) c] b=a b$.

Proof: Apply Theorem 2: $(a b) c=d,[(a b) c] a=d a=[(a b) c] b=d b=a b$. Q.E.D.

The theorem remains valid for $c=a$ or $c=b$ when $[(a b) a] a=[(a b) b] b=a b$.

The expression $(a b) a$ or $(a b) b$ cannot be simplified in the general case.

Theorem 7 (Figure 18): Symmetry of incidence relation: If equation $a x=b$ has at least one solution for $x$, then equation $b y=a$ has at least one solution for $y$.

Proof: If $a x=b$, choose $y=a b$; then $b(a b)=(a x)(a b)=a$.

Theorem 8 (Figure 20): The associative property $(a b) c=a(b c)=b(c a)$ does not hold.

Proof: I will prove that if two associative elements are equal, the third one cannot exist. Suppose that $(a b) c=a(b c), a \neq c, a b \neq b c$. By Theorem 3 $(a b) c=a(b c)=c a=(a b)(b c)=b$. This means that if $(a b) c=a(b c), a \neq c$, $a b \neq b c$, then $b=c a$, and $b(c a)$ does not exist. Q.E.D.

The equalities $(a b) c=(b c) a=(c a) b$ can only hold if $a b=b c=c a$. Otherwise, if two elements are equal from $(a b) c,(b c) a,(c a) b$, the third element does not exist.

\subsection{Other Properties in Subsection 2}

Figure 9: From $a b=a c$ does not follow $b=c$ in the general case.

Figure 12: Equation $u x=x$ has no solution for $x$ (no unit element $u$ exist for element $x$ ). This property cannot be derived from the axioms.

Figure 16: Spherical representation of the incidence relation by a point on a line, or two perpendicular lines, or two "perpendicular points" at $90^{\circ}$ distance from each other.

Figure 17: The inverse operation connected to the incidence relation on the spherical model. If equation $a x=b$ has at least one solution for $x$, then $a$ and $b$ are incident; if there is no solution for $x$, then $a$ and $b$ are not incident.

Figure 19: One of the possible options of displaying an associative element $(a b) c$ in spherical geometry.

Figure 21: The products of two associative elements are the same, shown as the orthocentre of the spherical triangle:

$[(a b) c][(b c) a]=[(b c) a][(c a) b]=[(c a) b][(a b) c]$. This property cannot be derived from the axioms. 


\subsection{Models of Projective Spheres}

Model 1 consists of three elements of symmetric roles. In the operation (Cayley) table the main diagonal is empty due the "aa undefined" property. The product of two different elements is the third element. No solution for the equation $u x=x$. I call this set a Klein projective sphere or K-sphere because it is similar to the Klein group deprived of the unit element.

Another form of displaying the set is the incidence table. I call an element in the last row the title element of the column above it which contains all elements incident to the title element below the column. If element $a$ is in the column of element $b$, then $b$ is in the column of a.The product of any two elements above a title element is the title element.

Model 2: "Ruin" the operation table of Model 1 by interchanging $b$ and $c$ among the products. Commutative property remains valid, and the main axiom also holds: $(a b)(a c)=b c=a ;(a b)(b c)=b a=b ;(a c)(b c)=c a=c$.

However, the roles of the three elements are not symmetric, and $u x=x$ does have solutions for $x$ in this set: $a b=b ; a c=c$.

An element $x$ is reflexive if there is at least one solution of equation $u x=x$ (The names "self-conjugated" or "self-incident" are also used).

Elements $a b=b$ and $a c=c$ are reflexive in this model, while $a$ is non-reflexive.

Table 1 and Table 2 show a Klein sphere displayed in an operation table and an incidence table. Table 3 and Table 4 show a Galois sphere of three elements with two reflexive (self-incident) elements also in an operation table and an incidence table.

Model 3: A set of seven elements:

Check the two axioms:

In the operation table (Table 5) the commutative property is shown by the symmetry to the main diagonal. The main axiom is tedious to check:

$(24)(27)=13=2$; etc. Apart from the empty main diagonal, each of the northwest-southeast diagonals in consists of all the elements numbered in reverse order. Elements 1, 5, 6 are reflexive, while 2, 3, 7 give a $\mathrm{K}$-sphere. The set is the equivalent of the Fano plane in classical projective geometry, so it is a Fano sphere.

In the incidence table (Table 6) the last row contains the title elements in the reverse order. The product of two elements can be determined in two ways: Find the title element of the only column that contains the two elements together; or, Find the common element of the two columns whose title elements are the two factors.

Model 4: A set of 13 elements:

The number of reflexive elements in a Galois sphere is the same as the number of elements incident to one and the same element, that is, the number of elements in the column above the title element. Table 7 shows a Galois sphere, reflexive elements shown in italics, as with Table 4 and Table 6. 
Table 1. Klein sphere in an operation table.

\begin{tabular}{llll}
\hline & $a$ & $b$ & $c$ \\
\hline$a$ & - & $c$ & $b$ \\
$b$ & $c$ & - & $a$ \\
$c$ & $b$ & $a$ & - \\
\hline
\end{tabular}

Table 2. Klein sphere in an incidence table.

\begin{tabular}{lll}
\hline 1 & 2 & 3 \\
2 & 3 & 1 \\
\hline 3 & 1 & 2 \\
\hline
\end{tabular}

Table 3. Galois sphere in an operation table.

\begin{tabular}{llll}
\hline & $a$ & $b$ & $c$ \\
\hline$a$ & - & $b$ & $c$ \\
$b$ & $b$ & - & $a$ \\
$c$ & $c$ & $a$ & - \\
\hline
\end{tabular}

Table 4. Galois sphere in an incidence table.

\begin{tabular}{lll}
\hline 1 & 2 & 3 \\
2 & 3 & 1 \\
\hline 1 & 3 & 2 \\
\hline
\end{tabular}

Table 5. Fano sphere in an operation table.

\begin{tabular}{llllllll}
\hline & 1 & 2 & 3 & 4 & 5 & 6 & 7 \\
\hline 1 & - & 1 & 2 & 1 & 4 & 4 & 2 \\
2 & 1 & - & 7 & 1 & 7 & 3 & 3 \\
3 & 2 & 7 & - & 6 & 7 & 6 & 2 \\
5 & 1 & 1 & 6 & - & 5 & 6 & 5 \\
6 & 4 & 7 & 7 & 5 & - & 4 & 5 \\
7 & 4 & 3 & 6 & 6 & 4 & - & 3 \\
\hline
\end{tabular}

Table 6. Fano sphere in an incidence table.

\begin{tabular}{ccccccc}
\hline 1 & 2 & 3 & 4 & 5 & 6 & 7 \\
2 & 3 & 4 & 5 & 6 & 7 & 1 \\
4 & 5 & 6 & 7 & 1 & 2 & 3 \\
\hline 1 & 7 & 6 & 5 & 4 & 3 & 2
\end{tabular}

(Example: 3 and 5 occur in the same column of title element 7; the columns of title element 3 and title element 5 share element 7 , so $35=7$ ) (See also Coxeter [8]). 
Table 7. Galois sphere with four reflexives in an incidence table.

\begin{tabular}{ccccccccccccc}
\hline 1 & 2 & 3 & 4 & 5 & 6 & 7 & 8 & 9 & 10 & 11 & 12 & 13 \\
2 & 3 & 4 & 5 & 6 & 7 & 8 & 9 & 10 & 11 & 12 & 13 & 1 \\
4 & 5 & 6 & 7 & 8 & 9 & 10 & 11 & 12 & 13 & 1 & 2 & 3 \\
10 & 11 & 12 & 13 & 1 & 2 & 3 & 4 & 5 & 6 & 7 & 8 & 9 \\
\hline 1 & 13 & 12 & 11 & 10 & 9 & 8 & 7 & 6 & 5 & 4 & 3 & 2 \\
\hline
\end{tabular}

Model 5: Any polarity between points and lines that satisfies the condition of polar reciprocity: If point $A$ fits line $b$, pole point $B$ fits polar line $a$. It can be constructed by a pole and polar defined by a planar conic, or a pair of pole points and their polar defined by a spherical conic. Antipolarity: the original polar reflected through the centre of the conic. Natural polarity of Figure 1 can be viewed as an antipolarity generated by a spherical circle of radius $45^{\circ}$.

Model 6: Ordered triples of elements of a field, (000) excluded, and $\left(a_{1}, a_{2}, a_{3}\right)=\left(l a_{1}, l a_{2}, l a_{3}\right) ; l \neq 0$. The product $\left(x_{1}, x_{2}, x_{3}\right)$ of elements $\left(a_{1}, a_{2}, a_{3}\right)$ and $\left(b_{1}, b_{2}, b_{3}\right)$ is given by an indeterminate system of two linear equations:

$$
\begin{aligned}
& a_{1} x_{1}+a_{2} x_{2}+a_{3} x_{3}=0, \\
& b_{1} x_{1}+b_{2} x_{2}+b_{3} x_{3}=0 .
\end{aligned}
$$

Model 7: Pencil of Euclidean straight lines through a point in 3D space [6]. The product of two lines is their common perpendicular in the pencil (Cf. vectorial product).

Model 8: Real and ideal points and real straight lines on the hyperbolic surface (Section Two).

Model 9: The Cartesian coordinate system of the plane with twofold polarity (Section Three).

\subsection{Theorems about Reflexive Elements}

( $x$ is reflexive if $u x=x$ has at least one solution.)

Theorem 9: Two reflexive elements cannot be incident to each other (In the column of a reflective element there is no other reflective element).

Proof: If $a b=a, b c=b$, then $a b=a=(a b)(b c)=b$ which is a contradiction. Q.E.D.

We do not need to state that $a \neq b, a \neq c, b \neq c$, because $a b=a$ gives $a \neq b ; \quad b c=b$ gives $b \neq c$; and $a=c$ would give $a b=a=b c=b a=b$ which contradicts to $a b=a$.

This is a fundamental property with important consequences for the structure of a finite or infinite projective sphere that contains reflexive elements.

Theorem 10: If two non-reflective elements are incident to a reflective element, they cannot be incident to each other (If two non-reflectives in the incidence table are in the column of a reflective element, they cannot occur in each other's columns).

Proof: If $a b=a, a c=(b c) b=c \quad(a$ is reflexive, $b$ and $c$ are incident to $a, b$ 
incident to $c$ ), then $a=a b=a c=b c ; c=(b c) b=a b=a$ which is a contradiction. Q.E.D.

\subsection{Connection of Projective Spheres with Projective Planes}

Many theorems and proofs in the theory of projective planes can be formulated and proved for projective spheres, such as: Consequences of the existence of a triangle or a quadrilateral in the set; Theorems on perspectivity and projectivity; Number of elements in finite non-trivial sets [9]; Theorem of Gleason on Fano planes [10]; Theorem of Bose on ovals; Theorem of Baer on polarities, $n$-chains, $\mathrm{n}$-cycles, and number of reflexive elements; Theorem of Bruck and Ryser on subsets; etc.

\subsection{Change of Notation}

One of the greatest obstacles to the acceptance of a new theory is a new type of notation. Grassmann used a new symbol in his epoch-marking work that made his thoughts unaccessible for most of his contemporaries. Still, I take the risk, because I agree with Struik's remark [11] on the development of mathematics: "The improvement in technique was a result of the improvement in notation".

The binary operation in projective spheres is not associative: $(a b) c \neq a(b c)$ in the general case. The term $a b c$ is therefore meaningless in this form. A complex expression involves a forest of parentheses and brackets which make the notation awkward and confusing.

It took me decades to realize that change was necessary, and another couple of years to get used to my own reforms. However, once getting used to the new notation, I found that the benefits outweighed the inconveniences.

In what follows, the operations are performed strictly from the left to the right. Any variance from this order is indicated by vertical bars. There is no bar between the factors for the strongest priority; one bar for the less strong priority; two bars for the next, even less strong priority, and so on. It is often useful to rearrange the order of the elements to reach minimal number of bars. The expressions in the present paper contain at most three bars between two factors.

Examples:

The meaning of expression 1234 with traditional brackets is $1234=[(12) 3] 4$.

The following expressions are equal by the bar notation: $1234=2134=3|12| 4=3|21| 4=4|123=4| 213=4|| 3|12=4||3| 21$.

Another example that will be used later: $61|25| 4|45 ; \quad A=61| 25 \mid 4, B=45=54$, $A B=61|25| 4|45=61| 25|4| 54=4$.

Counter-examples: $1234 \neq 12 \mid 34 ; 1234 \neq 1324 ; 1234 \neq 1243$.

\subsection{Some Noteworthy Configurations}

Suppose that the expressions are defined. For example, 123|231 implies that 1, 2, 3 are all different, $12 \neq 3,23 \neq 1,123 \neq 231$.

Figure 22 shows the three concurrent altitudes and the orthocentre of the 
triangle: $123|231=231| 312=312 \mid 123=H$. The Fano configuration is: $12|34||13| 24=13|24||14| 23=14|23||12| 34=F$. Example of a Fano configuration in Table 8: Vertices of the quadrilateral: 2, 3, 4, 7; sides: $23=7$; $47=5 ; 24=1 ; 37=2 ; 27=3 ; 34=6$; diagonal points: $23 \mid 47=5$; $24|37=1 ; 27| 34=6$; diagonal points are collinear: $51=16=65=4$.

Figure 23 shows the Pappos configuration in a premissa-conclusion form: If $a_{1} a_{2}=a_{2} a_{3}=a_{3} a_{1} ; b_{1} b_{2}=b_{2} b_{3}=b_{3} b_{1}$; then: $a_{1} b_{2}\left|a_{2} b_{1}\left\|a_{2} b_{3}\left|a_{3} b_{2}=a_{2} b_{3}\right| a_{3} b_{2}\right\| a_{1} b_{3}\right| a_{3} b_{1}=a_{1} b_{3}\left|a_{3} b_{1} \| a_{1} b_{2}\right| a_{2} b_{1}=P$.

Figure 24 shows that this configuration can be built on five independent elements: $\begin{aligned} & 13|24||14| 23|||13| 25|| 15|23=13| 25|| 15|23||14| 25|| 15 \mid 24 \\ & =4|25||15| 24|||13| 24|| 15 \mid 24=P\end{aligned}$

Figure 25 denotes the vertices of two perspective triangles of the Desargues configuration: If $a_{1} b_{1}\left|a_{2} b_{2}=a_{2} b_{2}\right| a_{3} b_{3}=a_{3} b_{3} \mid a_{1} b_{1}$, then: $a_{1} a_{2}\left|b_{1} b_{2}\left\|a_{1} a_{3}\left|b_{1} b_{3}=a_{1} a_{3}\right| b_{1} b_{3}\right\| a_{2} a_{3}\right| b_{2} b_{3}=a_{2} a_{3}\left|b_{2} b_{3} \| a_{1} a_{2}\right| b_{1} b_{2}=D$.

Figure 26 displays one triangle denoted by vertices, the other by sides: $a_{1} b_{1} c_{2}\left|b_{1} c_{1} a_{2}=b_{1} c_{1} a_{2}\right| c_{1} a_{1} b_{2}=c_{1} a_{1} b_{2} \mid a_{1} b_{1} c_{2}$ gives $a_{2} b_{2} c_{1}\left|b_{2} c_{2} a_{1}=b_{2} c_{2} a_{1}\right| c_{2} a_{2} b_{1}=c_{2} a_{2} b_{1} \mid a_{2} b_{2} c_{1}=D$.

Figure 27 demonstrates that the Desargues configuration needs six elements. Five elements are independent, the sixth element is only for generating element 16 (The structure remains the same if element 6 is moving anywhere along the product 16$)$. With this notation, we have:

$61|23| 4|51| 2|34=16| 54|3| 21|5| 43=61|23| 4|51| 2|| 16|54| 3|21| 5=D$.

The Little-Desargues configuration (Figure 28) is derived from the third definition of the Desargues configuration (Figure 27) replacing " 16 " or "61" by 1 , and " 1 " by 341 . For example:

“61” | 23 | 4 | 5“1” | 2 | $34=1 \mid 23$ | 4 || 5|341| 2 |34 = 2314|3415| 2 |34. Element 1 is shown as a line, elements 2, 3, 4, 5 are points in Figure 28.

Table 8. Fano configuration.

\begin{tabular}{lllllll}
\hline 1 & 2 & 3 & 4 & 5 & 6 & 7 \\
2 & 3 & 4 & 5 & 6 & 7 & 1 \\
4 & 5 & 6 & 7 & 1 & 2 & 3 \\
\hline 1 & 7 & 6 & 5 & 4 & 3 & 2 \\
\hline
\end{tabular}

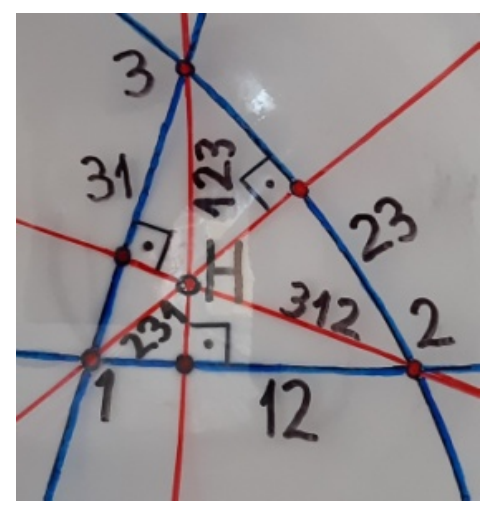

Figure 22. Hesse-Chasles configuration. 


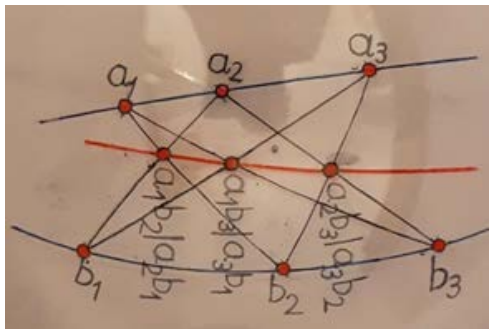

Figure 23. Pappos configuration (Definition 1).

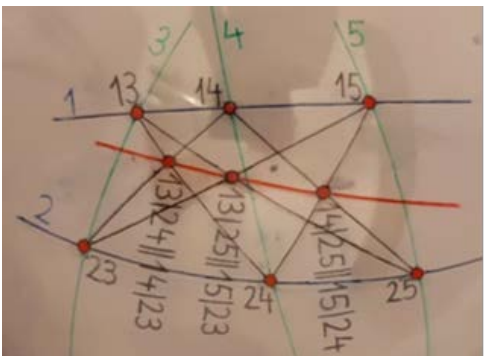

Figure 24. Pappos configuration (Definition 2).

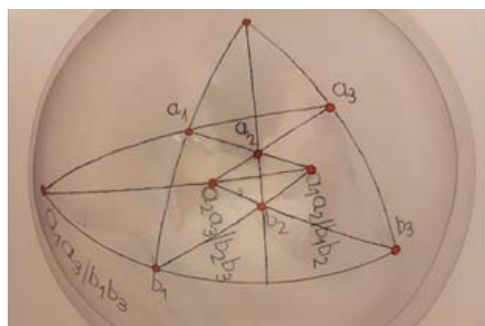

Figure 25. Desargues configuration (Definition 1).

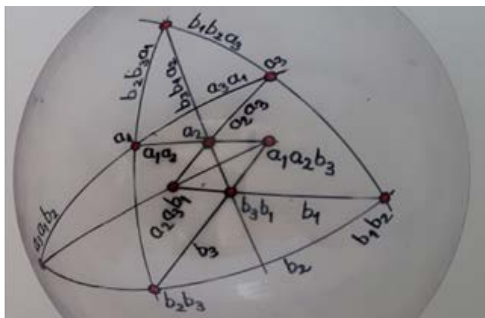

Figure 26. Desargues configuration (Definition 2).

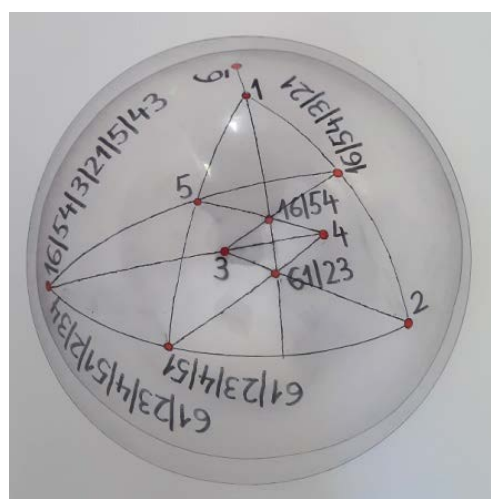

Figure 27. Desargues configuration (Definition 3). 


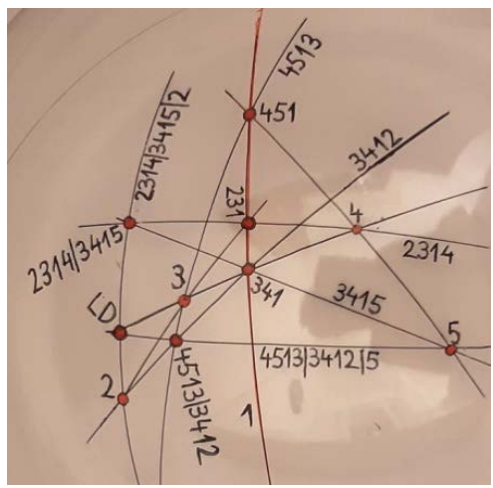

Figure 28. Little-Desargues configuration.

\subsection{Proving Theorems}

Following are some examples of applying the algebra of projective spheres on the equivalents of classical theorems in projective planes. The proof shows the three steps that many other proofs follow in projective spheres and projective planes (cf. Hilbert's proofs about Desarguesian planes in [9]). First step: Lemma. Second step: Dual of the lemma. Third step: Final proof, based on the two dual lemmas.

The following proofs make extended use of Theorem 2 using the short form $12=13$ instead of $12=13=23$, Theorem 4 using $12=34$ instead of $12=34=13=24=14=23$, and Theorem 7 about simplyfing an expression $(1231=1232=12)$.

\subsection{Theorem of Hessenberg}

Theorem 11: A Pappos sphere is a Desargues sphere.

Assumption: Given a Pappos sphere (in which the Pappos configuration is added as the third axiom to the two axioms in subsection 3), prove that it is also a Desargues sphere.

Following is Hessenberg's proof [12] translated to the algebra of the present system.

Apply the first form of Pappos configuration (Figure 23) according to the following pattern:

\begin{tabular}{ccc}
\hline$a_{1}$ & $a_{2}$ & $a_{3}$ \\
$b_{1}$ & $b_{2}$ & $b_{3}$ \\
$a_{2} b_{3} \mid a_{3} b_{2}$ & $a_{1} b_{3} \mid a_{3} b_{1}$ & $a_{1} b_{2} \mid a_{2} b_{1}$ \\
\hline
\end{tabular}

Premise: $a_{1} a_{2}=a_{2} a_{3}=a_{3} a_{1}, \quad b_{1} b_{2}=b_{2} b_{3}=b_{3} b_{1}$,

Conclusion:

$a_{1} b_{2}\left|a_{2} b_{1}\left\|a_{2} b_{3}\left|a_{3} b_{2}=a_{2} b_{3}\right| a_{3} b_{2}\right\| a_{1} b_{3}\right| a_{3} b_{1}=a_{1} b_{3}\left|a_{3} b_{1} \| a_{1} b_{2}\right| a_{2} b_{1}$.

Apply Definition 3 of the the Desargues configuration (Figure 27):

$61|23| 4|51| 2|34=16| 54|3| 21|5| 43=61|23| 4|51| 2|| 16|54| 3|21| 5=D$.

Another form, by multiplying all sides of the chain of equations by 3 or 4 : 


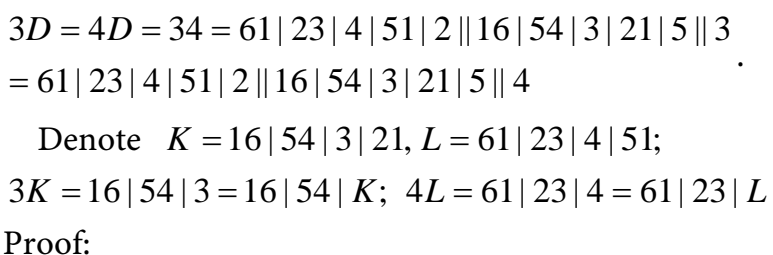

First Pappos configuration:

\begin{tabular}{ccc}
\hline $61 \mid 23$ & 2 & $23 \mid 45$ \\
5 & 1 & $L$ \\
$23|45| 1 \mid 2 L$ & 4 & $61 \mid 25$ \\
\hline
\end{tabular}

Conclusion: $23|45| 1|2 L||61| 25=61|25| 4$ (a complex expression converted to a simpler form).

Second Pappos configuration:

\begin{tabular}{ccc}
\hline $16 \mid 54$ & 5 & $23 \mid 45$ \\
2 & 1 & $K$ \\
$23|45| 1 \mid 5 K$ & 3 & $16 \mid 25$ \\
\hline
\end{tabular}

Conclusion: $23|45| 1|5 K \| 16| 25=16|25| 3$ (a complex expression converted to a simpler form).

Third Pappos configuration:

\begin{tabular}{ccc}
\hline $23|45| 1 \mid 5 K$ & $23 \mid 45$ & $23|45| 1 \mid 2 L$ \\
2 & $16 \mid 25$ & 5 \\
$23|45| 1|2 L||16| 25|| 45$ & $5 K \mid 2 L$ & $23|45| 1|5 K||16| 25|| 23$ \\
\hline
\end{tabular}

Conclusion:

$23|45| 1|5 K||16| 25|| 23|| 5 K|2 L=23| 45|1| 2 L|| 16|25||45||5 K| 2 L$

(Apply the results of the first and second Pappos configurations to simplify the result of the third Pappos configuration into a shorter form that proves the theorem.)

$$
\begin{aligned}
& =16|25| 3|23 \| 5 K| 2 L=5 K|2 L| 3=5 K|2 L||61| 25|4| 45=5 K|2 L| 4 \\
& =34=(\text { replacing } K=16|54| 3|21, L=61| 23|4| 51) \\
& =61|23| 4|51| 2 \| 16|54| 3|21| 5|| 3=61|23| 4|51| 2|| 16|54| 3|21| 5|| 4
\end{aligned}
$$

Multiply both sides by $5 K$ or $2 L$ :

$$
\begin{aligned}
& 2 L|5 K=2 L| 34=5 K|34=61| 23|4| 51|2||16| 54|3| 21 \mid 5 \\
& =61|23| 4|51| 2|34=16| 54|3| 21|5| 43
\end{aligned}
$$

Q.E.D.

Remark: The proof shows that the Hessenberg theorem applies to all finite or infinite Pappos spheres, i.e. a projective sphere with the two basic axioms and the Pappos property as the third axiom. 


\subsection{The Hesse-Chasles Configuration}

If expressions $123|231 ; 231| 312 ; 312 \mid 123$ are defined, they are concurrent:

$123|231=231| 312=312 \mid 123=H$. Geometrically it corresponds to the three altitudes and the orthocentre of a triangle (Figure 21).

A system which is built on two dual sets of "points" and "lines" does not deal with the product of a "point" and a "line", two elements taken from two different sets. The projective spheres are built on one set of elements. Therefore the product of a "point" and a "line" is also defined, for example, as a perpendicular dropped from the point to the line.

The Hesse-Chasles or Hesse configuration only requires three elements, in contrast with the four independent elements of the Fano configuration, or five elements of Pappos, or six elements of the Desargues configuration.

Many classical algebraic systems use axioms involving three elements, such as semigroups, Clifford algebras, Lie-Killing or Jordan algebras, Gatial algebra, etc. [13]. In like manner, we can study projective spheres with the Hesse configuration as the third axiom to the two basic axioms. An algebraic system with only three elements used in the axioms may prove to be easier to handle than another system of axioms with five or six defining elements.

Following are two simple theorems about well-known geometric facts in a Hesse sphere, that is, in a projective sphere endowed with the Hesse configuration as the third axiom in the set.

\subsection{Theorem about Quadrilaterals in a Hesse Sphere}

Theorem 12: For any four elements, the products $12 \mid 34,1234,1243$ are concurrent:

$$
12|34| 1234=1234|1243=12| 34 \mid 1243
$$

The proof is simple:

$$
A=12, B=3, C=4 ; A B C=1234, B C A=34|12=12| 34, C A B=4|12| 3=1243
$$

Likewise, 34|12, 3412, 3421 are also concurrent:

$$
\begin{aligned}
& 34|12| 3412=3412|3421=34| 12 \mid 3421 \\
& \text { But } 34|12=43| 21=12 \mid 34, \text { so we have } \\
& \qquad \begin{array}{l}
12|34| 1234=1234|1243=1243||12| 34 \\
43|21| 4321=4321|4312=4312||43| 21
\end{array}
\end{aligned}
$$

This gives that element $12|34=43| 21$ is concurrent with elements 1234,1243 , and also with elements $4312,4321$.

\subsection{Theorem about Closure in a Hesse Sphere}

Theorem 13: If elements 1, 2, 3 determine their Hesse element: $123|231=231| 312=312 \mid 123=H$, then $12 H|2 H 1=2 H 1| H 12=H 12 \mid 12 H=3$ (Any three elements of the set $1,2,3, \mathrm{H}$ determine the fourth element. It is the generalization of a theorem in absolute 
geometry about the vertices and the orthocentre of a triangle).

Proof: (The clue is that from equalities $123|231=231| 312=12 \mid 123=H$ always choose the one that best fits the simplification of the given expression).

$$
\begin{gathered}
12 H=12 \| 123|312=312| 123 \mid 12=123 \\
1 H 2=1|| 123|231||2=123| 231|1| 2=231 \mid 2=23 \\
2 H 1=2\|123|231 \| 1=231| 312|2| 1=312 \mid 1=13
\end{gathered}
$$

Now $12 H|2 H 1=2 H 1| H 12=H 12|12 H=123| 23=23|13=123| 23=3$. Q.E.D.

\subsection{Two Non-Isomorphic Projective Spheres with 21 Elements}

The theory of finite projective planes admits only one projective plane with 21 points and 21 lines. In contrast, there are two non-isomorphic projective spheres with 21 elements shown in Table 9 and Table 10. Their incidence tables are isomorphic, but the title elements in the last row are arranged differently [6] [7]. The Fano property applies both sets, but the Hesse property only in the first.

Triangle with an orthocentre (Table 9), and another triangle without an orthocentre (Table 10):

Table 9: Elements (“triangle"): 1, 3, 18; associative elements (“altitudes"): $13(18)=9 ; 3(18) 1=2 ;(18) 13=13$ are concurrent: $92=2(13)=(13) 9=(14)$ (“orthocentre”).

Table 10: Elements ("triangle”): 1, 3, (18); associative elements (“altitudes"): $13(18)=8 ; 3(18) 1=2 ;(18) 13=(19)$ are not concurrent: $2 \neq 2(19) \neq(19) 8 \neq 82$ (“orthocentre" does not exist).

Table 9. Galois sphere with five reflexives.

\begin{tabular}{ccccccccccccccccccccc}
\hline 1 & 2 & 3 & 4 & 5 & 6 & 7 & 8 & 9 & 10 & 11 & 12 & 13 & 14 & 15 & 16 & 17 & 18 & 19 & 20 & 21 \\
2 & 3 & 4 & 5 & 6 & 7 & 8 & 9 & 10 & 11 & 12 & 13 & 14 & 15 & 16 & 17 & 18 & 19 & 20 & 21 & 1 \\
5 & 6 & 7 & 8 & 9 & 10 & 11 & 12 & 13 & 14 & 15 & 16 & 17 & 18 & 19 & 20 & 21 & 1 & 2 & 3 & 4 \\
15 & 16 & 17 & 18 & 19 & 20 & 21 & 1 & 2 & 3 & 4 & 5 & 6 & 7 & 8 & 9 & 10 & 11 & 12 & 13 & 14 \\
17 & 18 & 19 & 20 & 21 & 1 & 2 & 3 & 4 & 5 & 6 & 7 & 8 & 9 & 10 & 11 & 12 & 13 & 14 & 15 & 16 \\
\hline 1 & 21 & 20 & 19 & 18 & 17 & 16 & 15 & 14 & 13 & 12 & 11 & 10 & 9 & 8 & 7 & 6 & 5 & 4 & 3 & 2
\end{tabular}

Table 10. Non-Galois sphere with nine reflexives.

\begin{tabular}{ccccccccccccccccccccccc}
\hline 1 & 2 & 3 & 4 & 5 & 6 & 7 & 8 & 9 & 10 & 11 & 12 & 13 & 14 & 15 & 16 & 17 & 18 & 19 & 20 & 21 \\
2 & 3 & 4 & 5 & 6 & 7 & 8 & 9 & 10 & 11 & 12 & 13 & 14 & 15 & 16 & 17 & 18 & 19 & 20 & 21 & 1 \\
5 & 6 & 7 & 8 & 9 & 10 & 11 & 12 & 13 & 14 & 15 & 16 & 17 & 18 & 19 & 20 & 21 & 1 & 2 & 3 & 4 \\
15 & 16 & 17 & 18 & 19 & 20 & 21 & 1 & 2 & 3 & 4 & 5 & 6 & 7 & 8 & 9 & 10 & 11 & 12 & 13 & 14 \\
17 & 18 & 19 & 20 & 21 & 1 & 2 & 3 & 4 & 5 & 6 & 7 & 8 & 9 & 10 & 11 & 12 & 13 & 14 & 15 & 16 \\
1 & 4 & 3 & 18 & 11 & 15 & 21 & 17 & 16 & 19 & 6 & 13 & 12 & 20 & 10 & 9 & 8 & 5 & 14 & 7 & 2 \\
\hline
\end{tabular}




\subsection{Projective Spheres with 91 Elements}

Devidé [14] proved formulas for the possible number of reflexive elements. Applying his results, a projective sphere with 91 elements may have $10,16,22$, or 28 reflexive elements. I constructed all four of them.

The sphere with 10 reflectives admits the Hesse, Pappos and consequently the Desargues properties but not the Fano property. The sphere with 28 reflectives admits the Pappos, consequently the Desargues properties but not the Hesse or Fano properties. The spheres with 16 and 22 reflectives built on Dickson quasifield admit none of these properties [15] [16].

I found other possible distribution of reflective elements in spheres with 16 and 22 reflectives, but I was unable to construct these spheres or refute their existence.

\section{The Hyperbolic Surface as a Model of Projective Spheres (Hyperbolic Napier Pentagon vs. Spherical Napier Pentagram)}

\subsection{What Type of Line in Planar, Spherical, or Hyperbolic Geometry Corresponds to the Transversal of Two Skew Straight Lines in Euclidean 3D Space?}

Gábor Gévay and Lajos Szilassi [17] drew my attention to the classic theorem of Petersen and Morley [18] about skew straight lines in Euclidean 3D space. Given three skew straight lines, their three common perpendiculars fit one and only one common perpendicular. The resulting structure is perfectly symmetrical for each of the ten straight lines, reminiscent of the ten points or lines in the $2 \mathrm{D}$ Desargues configuration (Figure 29).

My question was if a similar theorem about skew lines could be accomplished on a $2 \mathrm{D}$ surface. The Euclidean plane, the Menelaosian sphere (on which two opposite points are different) or the Riemannian sphere (on which two opposite points are indistinguishable) are out of question, because they do not admit skew straight lines. However, skew straight lines do exist in 2D hyperbolic geometry. Two hyperbolic skew lines (non-intersecting and non-parallel straight lines) determine one and only one common perpendicular. This line can be defined as the equivalent of the transversal of two skew lines in Euclidean space. It can also be called a transversal; but I keep to the more suggestive term "common perpendicular".

In this chapter, hyperbolic figures are mostly shown on the open hemisphere of the Poincaré model. Hyperbolic straight lines are open spherical semicircles perpendicular to the equatorial plane.

\subsection{Hyperbolic Rectangular Hexagon on 2D Surface as the Equivalent of Skew Euclidean Rectangular Hexagon in 3D Space}

Three Euclidean skew lines with three common perpendiculars form a skew 
hexagon in the Euclidean 3D space. Any two adjacent sides are perpendicular to each other. Three hyperbolic skew lines with three common perpendiculars form a hyperbolic convex hexagon on the hyperbolic 2D surface with six interior angles $90^{\circ}$ each (Figure 30 ).

\subsection{Hyperbolic Rectangular Hexagon vs. a Pair of Spherical Polar Triangles}

The hyperbolic rectangular hexagon can be interpreted as the hyperbolic counterpart of a pair of spherical polar triangles which determine a spherical rectangular hexagram with intersecting sides.

Figure 31 shows a spherical rectangular hexagram, Figure 32, a hyperbolic rectangular hexagon. The sequence of sides can be traced along the path $a-a b-b$ $b c-c-c a-a$ in both cases.

Figure 33 shows the vertices $P_{1}, P_{2}, P_{3}, P_{4}, P_{5}, P_{6}$ and sides $t_{12}=P_{1} P_{2}, t_{23}=P_{2} P_{3}$, $\mathrm{t}_{34}=\mathrm{P}_{3} \mathrm{P}_{4}, \mathrm{t}_{45}=\mathrm{P}_{4} \mathrm{P}_{5}, \mathrm{t}_{56}=\mathrm{P}_{5} \mathrm{P}_{6}, \mathrm{t}_{61}=\mathrm{P}_{6} \mathrm{P}_{1}$ of the spherical hexagram.

Figure 34 shows the vertices $\mathrm{P}_{1}, \mathrm{P}_{2}, \mathrm{P}_{3}, \mathrm{P}_{4}, \mathrm{P}_{5}, \mathrm{P}_{6}$ and sides $\mathrm{s}_{12}=\mathrm{P}_{1} \mathrm{P}_{2}, \mathrm{~s}_{23}=\mathrm{P}_{2} \mathrm{P}_{3}$, $\mathrm{s}_{23}=\mathrm{P}_{2} \mathrm{P}_{3}, \mathrm{~s}_{45}=\mathrm{P}_{4} \mathrm{P}_{5}, \mathrm{~s}_{45}=\mathrm{P}_{4} \mathrm{P}_{5}, \mathrm{~s}_{61}=\mathrm{P}_{6} \mathrm{P}_{1}$ of the hyperbolic hexagon. The points of intersection of non-adjacent sides are missing, because hyperbolic skew lines have no common points.

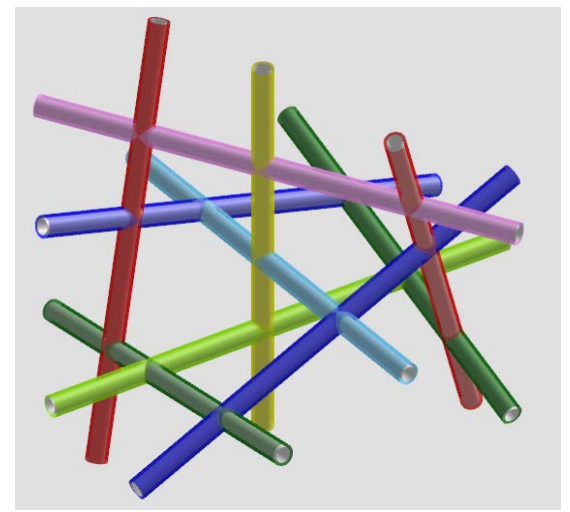

Figure 29. GeoGebra illustration of Szilassi to the Petersen-Morley theorem.

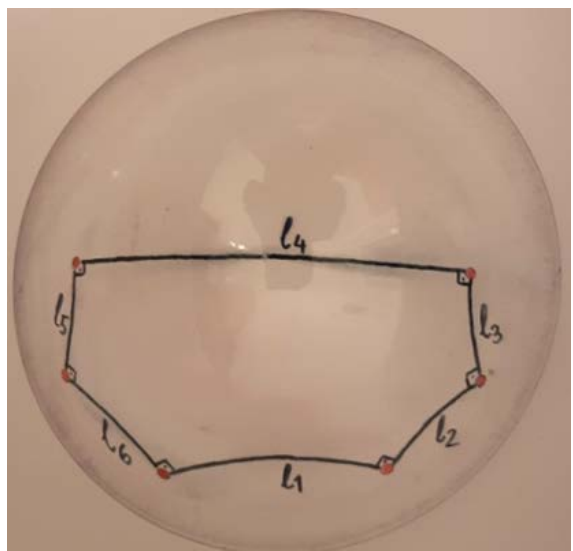

Figure 30. Hyperbolic convex hexagon with six interior right angles. 


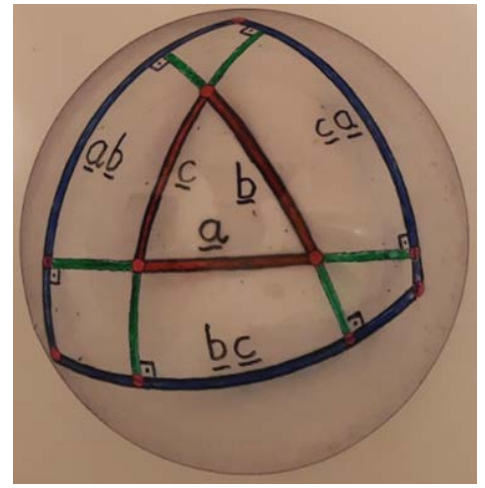

Figure 31. Spherical rectangular hexagram.

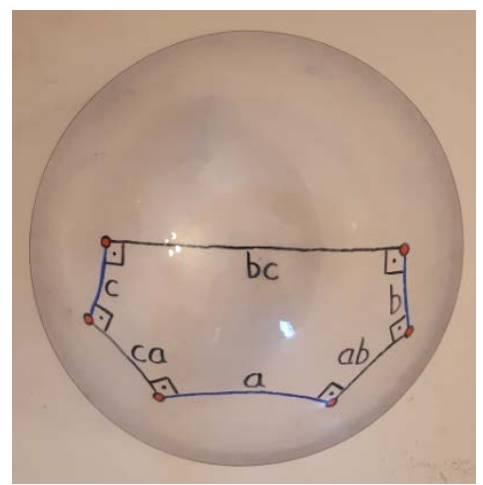

Figure 32. Hyperbolic rectangular hexagon.

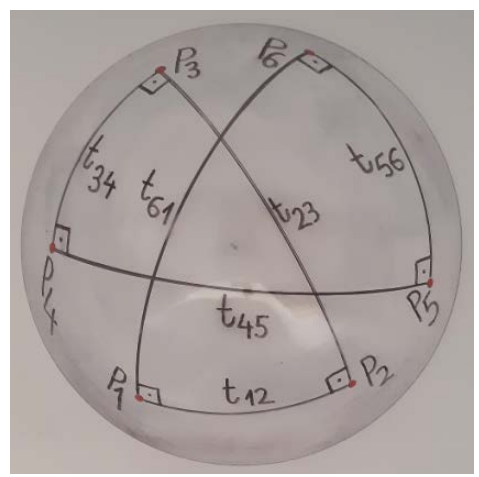

Figure 33. Vertices and sides of a spherical rectangualar hexagram.

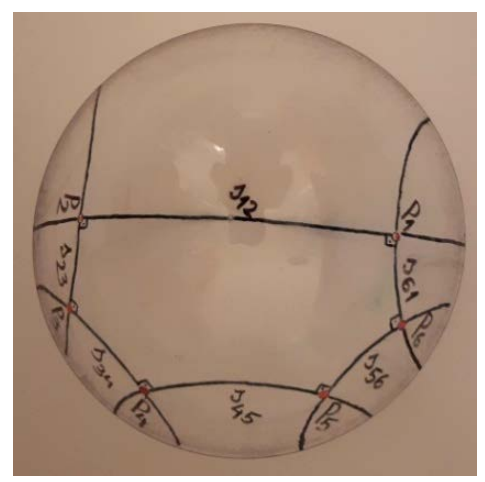

Figure 34. Vertices and sides of a hyperbolic rectangular hexagon. 
As seen in Figure 33 and Figure 34, the vertices of the spherical hexagram do not coincide with the vertices of the spherical polar triangles. The sides of the rectangular spherical hexagon correspond to the equatorial arcs of the angles of the polar triangles. For example, the angle at vertex $\mathrm{P}_{6} \mathrm{P}_{1} \mid \mathrm{P}_{2} \mathrm{P}_{3}$ corresponds to the equatorial $\operatorname{arc} \mathrm{P}_{1} \mathrm{P}_{2}$. Consequently, the sides of the hyperbolic rectangular hexagon correspond to the angles of spherical polar triangles.

\subsection{Hyperbolic Napier Pentagon with Five Right Angles}

The hyperbolic rectangular hexagon has six right angles. Are there hyperbolic rectangular polygons with fewer than six right angles?

A biangle with two right angles or a triangle with three right angles or a quadrilateral with four right angles cannot be constructed on the hyperbolic surface, but a hyperbolic convex pentagon with five right angles does exist, and corresponds to the spherical Napier pentagram.

Figure 35 shows a spherical rectangular Napier pentagram with right angles at each vertex $\mathrm{P}_{1}, \mathrm{P}_{2}, \mathrm{P}_{3}, \mathrm{P}_{4}, \mathrm{P}_{5}$. The points of intersection $\mathrm{P}_{1} \mathrm{P}_{2}\left|\mathrm{P}_{3} \mathrm{P}_{4}, \mathrm{P}_{2} \mathrm{P}_{3}\right| \mathrm{P}_{4} \mathrm{P}_{5}, \mathrm{P}_{3} \mathrm{P}_{4}\left|\mathrm{P}_{5} \mathrm{P}_{1}, \mathrm{P}_{4} \mathrm{P}_{5}\right| \mathrm{P}_{1} \mathrm{P}_{2}, \mathrm{P}_{5} \mathrm{P}_{1} \mid \mathrm{P}_{2} \mathrm{P}_{3}$ are the vertices of a spherical convex pentagon.

Figure 36 shows a hyperbolic rectangular Napier pentagon with right angles at each vertex $\mathrm{P}_{1}, \mathrm{P}_{2}, \mathrm{P}_{3}, \mathrm{P}_{4}, \mathrm{P}_{5}$. The points of intersection of non-adjacent sides are missing, because skew lines have no common points.

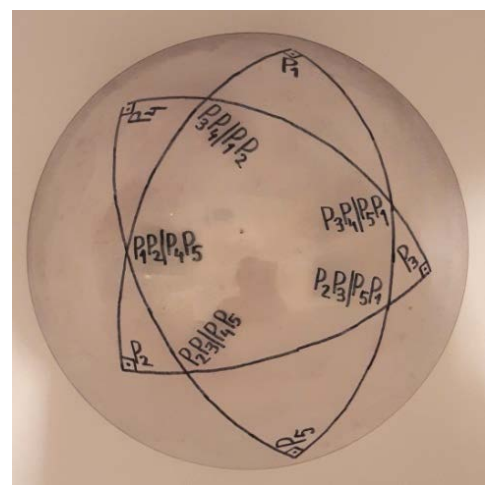

Figure 35. Spherical Napier pentagram.

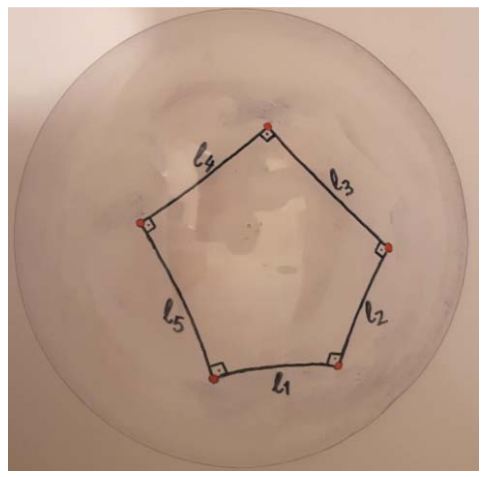

Figure 36. Hyperbolic Napier pentagon. 


\subsection{Cycles of Incidence on the Sphere}

The incidence relation was discussed in Section One (Figure 16).

An $m$-cycle of incidence is a series of elements in which any two adjacent elements are incident, including the last and the first (Cf. [1] [18]). In this sense, a point on a straight line or two perpendicular straight lines represent 2-cycles. Likewise, two perpendiculars and their point of intersection constitute a 3-cycle in any geometry. A spherical octant with three right angles or a spherical segment of length $90^{\circ}$ are both 3-cycles.

What are the spherical 5-cycles? A spherical Napier pentagram in Figure 37 is a 5 -cycle of elements $1,2,3,4,5$. Each element on the figure consists of a pole point and a polar equator. A spherical Lambert quadrilateral in Figure 38 is a 5-cycle of elements 1, 2, 3, 4 as lines, and element 5 as the point of intersection of lines 1 and 4 . Point 5 cannot be omitted from the cycle (jumping directly from line 4 to line 1), because lines 4 and 1 are not perpendiculars. The spherical triangle with one right angle is a 5-cycle of elements 1, 2, 4 as lines, elements 3, 5 as points (Figure 39). The vertex at the intersection of lines 1 and 2 is omitted from the cycle (jumping directly from line 1 to line 2), because lines 1 and 2 are perpendiculars.

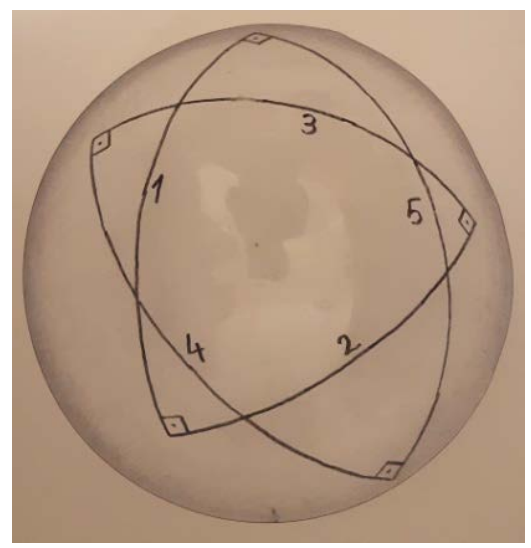

Figure 37. A 5-cycle as a spherical Napier pentagram.

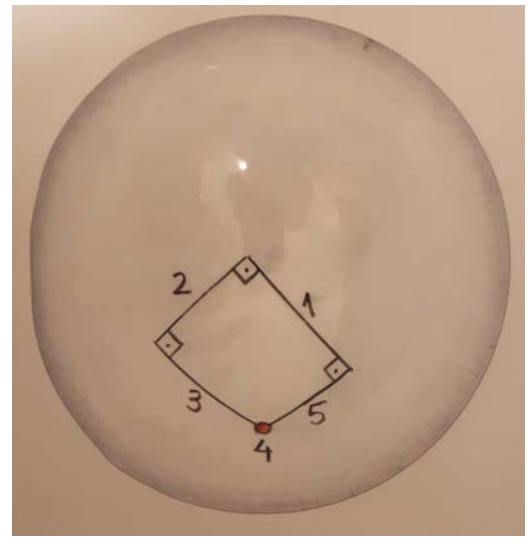

Figure 38. A 5-cycle as a spherical Lambert quadrilateral. 


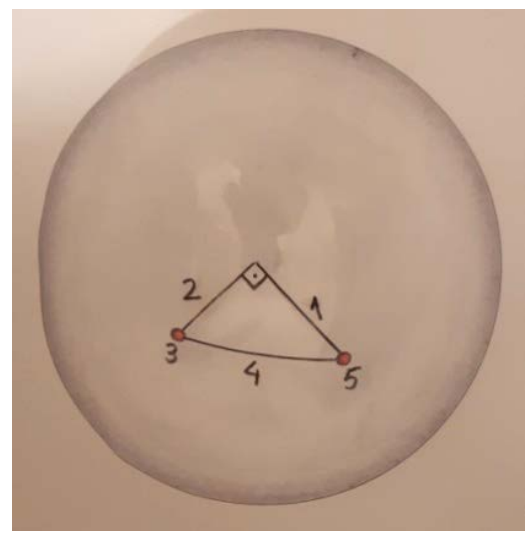

Figure 39. A 5-cycle as a right-angled spherical triangle.

\subsection{The Hyperbolic Surface as a Projective Sphere}

The infinite hyperbolic surface does not admit natural polarity in the form as it exists on the sphere (Figure 1). However, if we prove that the points and straight lines of the hyperbolic surface can be arranged into a model of the projective sphere, the concept of the cycles of incidence can be extended to the hyperbolic surface.

Consider the real and ideal points and the real straight lines of the hyperbolic surface (The ideal line is omitted from the construction). Prove that any two different elements in this set uniquely determines a resulting element in the same set.

1) Two real points, or two ideal points, or a real point and an ideal point determine a real straight line through the points.

2) Two intersecting real straight lines determine the point of intersection.

3) Two parallel real straight lines determine the common ideal point.

4) Two skew real straight lines determine the common perpendicular which is also a real straight line.

5) Whether or not the real point is on the real straight line, the point and the line determine the perpendicular dropped from the point to the line.

6) A real straight line and an ideal point not on the line determine the perpendicular dropped from the ideal point to the line.

7) In order to determine the result of an ideal point and a line through the ideal point, consider line $l$ and one of its ideal points $P$ (Figure 40). Moving from a real point on real straight line $I$ to ideal point $\mathrm{P}$, the figure suggests that the limiting case will be $\mathrm{P}$ itself. This is not a proof, just a way to visualize the process. Still, the only reasonable choice for the result of line $I$ and one of its ideal points $\mathrm{P}$ is $\mathrm{P}$ itself. The ideal point $\mathrm{P}$ is a reflexive element that can be viewed as either a point at infinity or a line degenerated into a point.

\subsection{Cycles of Incidence on the Hyperbolic Surface}

The arrangement of the hyperbolic surface into a projective sphere allows to apply cycles of incidence in the same manner as on the sphere. The incidence con- 
dition for a hyperbolic point and a straight line is that the point is on the line; for two straight lines is that they are perpendiculars; the only difference is that I was unable to extend the incidence relation to the case of two hyperbolic points.

A hyperbolic Napier pentagon in Figure 41 is a 5-cycle of elements 1, 2, 3, 4, 5 displayed as lines. A hyperbolic Lambert quadrilateral in Figure 42 is a 5-cycle of elements 1, 2, 3, 4 as lines, and element 5 the point of intersection of lines 1 and 4 . The hyperbolic triangle with one right angle is a 5 -cycle of elements 1, 2, 4 as lines, elements 3,5 as points (Figure 43).

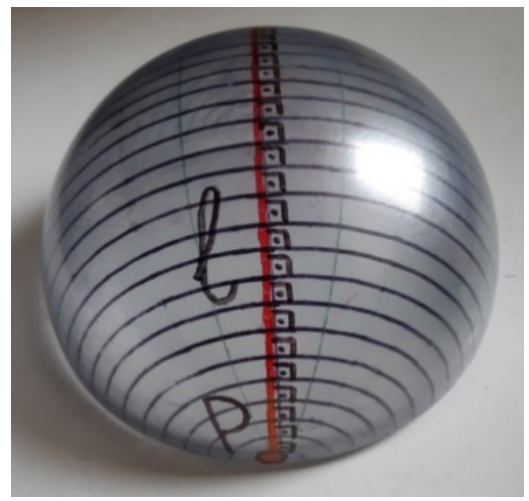

Figure 40. An ideal point as a reflective element.

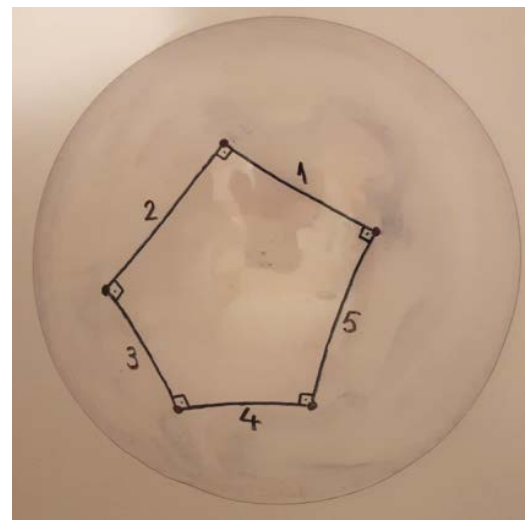

Figure 41. Hyperbolic Napier pentagon.

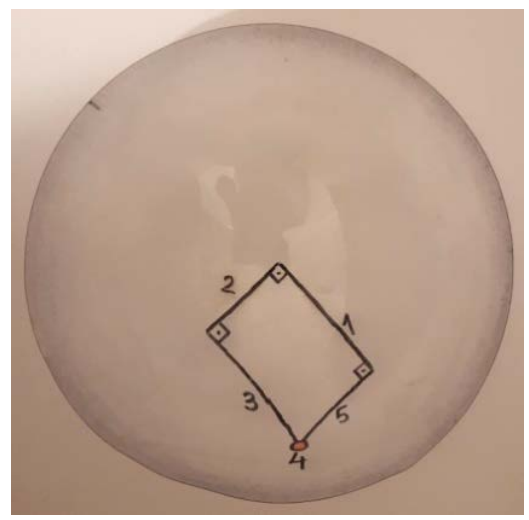

Figure 42. Hyperbolic Lambert quadrilateral. 


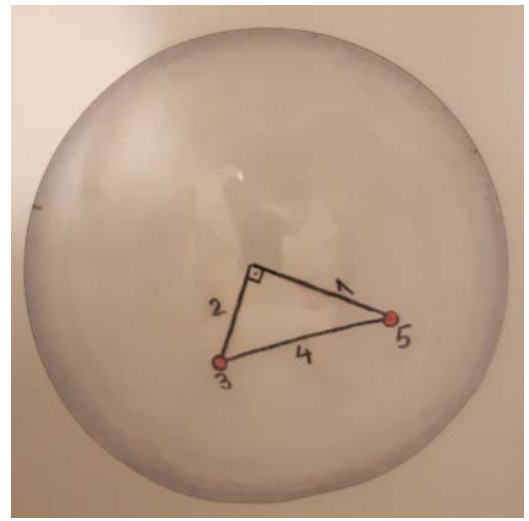

Figure 43. Hyperbolic right triangle.

\subsection{Hyperbolic 5-Cycles Are the Simplexes of Hyperbolic Geometry}

The 5-hyperbolic cycles, including the Napier pentagon and the Lambert quadrilateral, play a much more important role than just transferring certain polygons from the sphere to the hyperbolic surface. It is not the general triangle (which proves to be a 6-cycle), but the 5-cycle that can be viewed as the simplex of 2D hyperbolic geometry.

Hyperbolic geometry reveals the true meaning and significance of the Napier construction that was originally discovered 400 years ago in spherical geometry.

\subsection{The Fundamental Formula for the Hyperbolic Napier Pentagon}

Theorem 14: In a hyperbolic Napier pentagon any two adjacent sides $k, I$ determine the opposite side $n$ of the pentagon by the fundamental formula:

$$
\cosh \boldsymbol{n}=\sinh \boldsymbol{k} \sinh \boldsymbol{I}
$$

Figure 44 shows a right-angled triangle in the Napier pentagon with two adjacent sides denoted by $\operatorname{leg} 1$ and $\operatorname{leg} 2$ and their hypotenuse. In contrast, the Napier hypotenuse is the side of the pentagon opposite the hypotenuse defined by $\operatorname{leg} 1$ and $\operatorname{leg} 2$.

Figure 45 shows the fundamental formulas belonging to the hypotenuse and the Napier hypotenuse of the Napier pentagon: Denote $\operatorname{leg} 1=\boldsymbol{k}, \operatorname{leg} 2=\boldsymbol{l}$, hypotenuse $=\boldsymbol{m}$, Napier hypotenuse $=\boldsymbol{n}$, then: $\cosh \boldsymbol{m}=\cosh \boldsymbol{k} \cosh \boldsymbol{I}$, and $\cosh \boldsymbol{n}=\sinh \boldsymbol{k} \sinh \boldsymbol{l}$.

Draft of the proof (Figure 46): Prove the corresponding theorem for the spherical Napier pentagram, then apply Osborn's rule to convert the formula to the hyperbolic Napier pentagon.

Proof:

Several authors, including Gauss [19], studied the trigonometry of the spherical pentagon $A B C D E$ (Figure 46). However, the present proof relates to the spherical Napier pentagram $\mathrm{P}_{1} \mathrm{P}_{2} \mathrm{P}_{3} \mathrm{P}_{4} \mathrm{P}_{5}$, because the spherical pentagram is the counterpart of the hyperbolic Napier pentagon. 


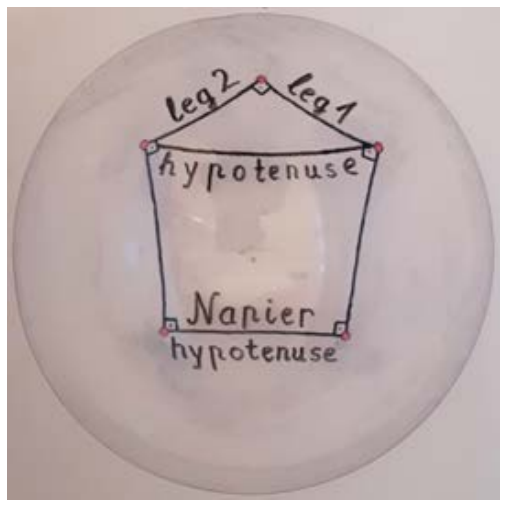

Figure 44. Napier pentagon and hypotenuses.

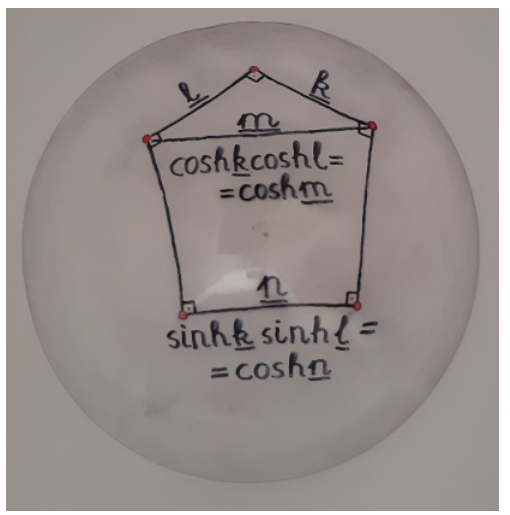

Figure 45. Fundamental formulas for the hypotenuses.

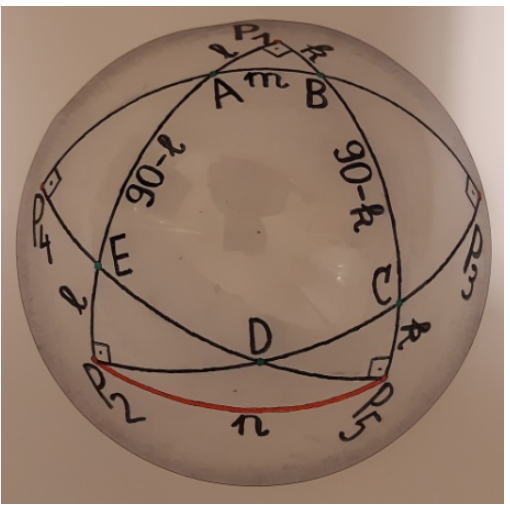

Figure 46. Draft of the proof of Theorem 25.

Given the right-angled triangle $A B \mathrm{P}_{1}$ with legs $A \mathrm{P}_{1}=\boldsymbol{I}, B \mathrm{P}_{1}=\boldsymbol{k}$, and hypotenuse $A B=\boldsymbol{m}$, we have $\cos \boldsymbol{m}=\cos \boldsymbol{k} \cos \boldsymbol{l}$. It is of advantage to use this formula in the quadratic form $\cos ^{2} \boldsymbol{m}=\cos ^{2} \boldsymbol{k} \cos ^{2} \boldsymbol{I}$ because both $\cos \boldsymbol{m}$ and $-\cos \boldsymbol{m}$ are correct solutions of the equation. Gauss also used the quadratic form $\frac{1}{\cos ^{2} \boldsymbol{k}}=\tan ^{2} \boldsymbol{C D} \tan ^{2} \boldsymbol{D E}$ for the pentagon $A B C D E$ (See also Coxeter's Frieze patterns [20]).

Geometrically, segment $A B$ and segment $\mathrm{P}_{3} \mathrm{P}_{4}$ both satisfy the equation, because $(-\cos \boldsymbol{m})=\cos (180-\boldsymbol{m})$. Here $-\cos \boldsymbol{m}=\cos (180-\boldsymbol{m})$ is relevant be- 
cause it refers to the segment between vertices $\mathrm{P}_{3}$ and $\mathrm{P}_{4}$, instead of the segment between $A$ and $B$ of the spherical pentagon $A B C D E$ (This property corresponds to the connection between the sides and the angles of a pair of spherical polar triangles).

Now consider the right-angled spherical triangle $\mathrm{P}_{5} \mathrm{P}_{1} \mathrm{P}_{2}$ with legs

$\mathrm{P}_{5} \mathrm{P}_{1}=90+\boldsymbol{k}, \quad \mathrm{P}_{2} \mathrm{P}_{1}=90+\boldsymbol{l}$, and hypotenuse $\mathrm{P}_{2} \mathrm{P}_{5}=\boldsymbol{n}$. Here $\cos ^{2} \boldsymbol{n}=\cos ^{2}(90+\boldsymbol{k}) \cos ^{2}(90+\boldsymbol{l})=\sin ^{2} \boldsymbol{k} \sin ^{2} \boldsymbol{I}$, so $\cos \boldsymbol{n}=-(\sin \boldsymbol{k} \sin \boldsymbol{l})$.

Note that $\boldsymbol{n}$ refers to the segment connecting two vertices of right angles of the spherical pentagram. The side of the hyperbolic Napier pentagon also connects two vertices of right angles, so Osborn's rule can be applied for the construction:

$$
\cosh \boldsymbol{n}=\sinh \boldsymbol{k} \sinh \boldsymbol{I} \text {. Q.E.D. }
$$

\subsection{Napier Pentagon Decomposed into Two Lambert Quadrilaterals}

Drop a perpendicular from a vertex of the Napier pentagon to the opposite side that divides the pentagon into two Lambert quadrilaterals. Conversely, any Lambert quadrilateral with angle $\alpha<90^{\circ}$ can be supplemented by another Lambert quadrilateral with angle $\left(90^{\circ}-\alpha\right)$ to form a Napier pentagon (Figure 47). This construction reminds of the decomposition of a spherical biangle into two supplementary triangles.

By notation of Figure 47 we have:

$$
\begin{gathered}
\tanh ^{2} \boldsymbol{c} \tanh ^{2} \boldsymbol{m}_{a}=\cos ^{2} \boldsymbol{a}_{1} \\
\tanh ^{2} \boldsymbol{d} \tanh ^{2} \boldsymbol{m}_{a}=\cos ^{2}\left(90-\boldsymbol{a}_{1}\right)=\sin ^{2} \boldsymbol{a}_{1}=1-\cos ^{2} \boldsymbol{a}_{1} \\
\tanh ^{2} \boldsymbol{c}+\tanh ^{2} \boldsymbol{d}=\frac{1}{\tanh ^{2} \boldsymbol{m}_{a}}
\end{gathered}
$$

\subsection{Hyperbolic Rectangular Hexagon Decomposed into Two Napier Pentagons}

The properties of the rectangular hexagon can be deduced from formula (1) of the Napier pentagon.

Figure 48 shows a hyperbolic rectangular hexagon with sides

$a=a_{1}+a_{2}=\mathrm{P}_{6} \mathrm{~F}_{61}+\mathrm{F}_{61} \mathrm{P}_{1}=\mathrm{P}_{6} \mathrm{P}_{1}, \quad b=\mathrm{P}_{1} \mathrm{P}_{2}, \quad c=\mathrm{P}_{2} \mathrm{P}_{3}$,

$d=d_{1}+d_{2}=\mathrm{P}_{3} \mathrm{~F}_{34}+\mathrm{F}_{34} \mathrm{P}_{4}=\mathrm{P}_{3} \mathrm{P}_{4}, \quad e=\mathrm{P}_{4} \mathrm{P}_{5}, \quad f=\mathrm{P}_{5} \mathrm{P}_{6}$.

The common perpendicular $m_{25}$ of sides $a, d$ decomposes the hexagon into two Napier pentagons with sides $m_{25}, a_{2}=\mathrm{F}_{61} \mathrm{P}_{1}, b, c, d_{1}=\mathrm{P}_{3} \mathrm{~F}_{34}$ and $m_{25}, d_{2}=\mathrm{F}_{34} \mathrm{P}_{4}, e, f, a_{1}=\mathrm{P}_{6} \mathrm{~F}_{61}$ respectively.

Applying formula (1) for both pentagons, we get

$$
\cosh m=\sinh b \sinh c=\sinh e \sinh f
$$

Formula (2) corresponds to the law of sines in spherical geometry: $\sin \beta \sin b=\sin a \sin \alpha$ for sides and angles of spherical triangles. On this basis, other formulas of trigonometry can be established for the hyperbolic rectangular hexagons, such as the law of cosines, Ceva's theorem, Menelaus's theorem, etc. 


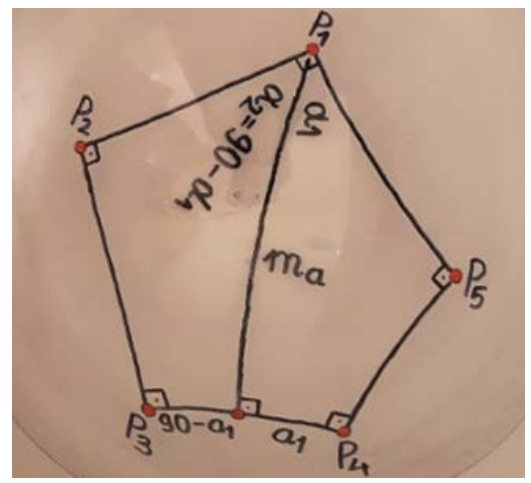

Figure 47. Napier pentagon decomposed into two Lambert quadrilaterals.

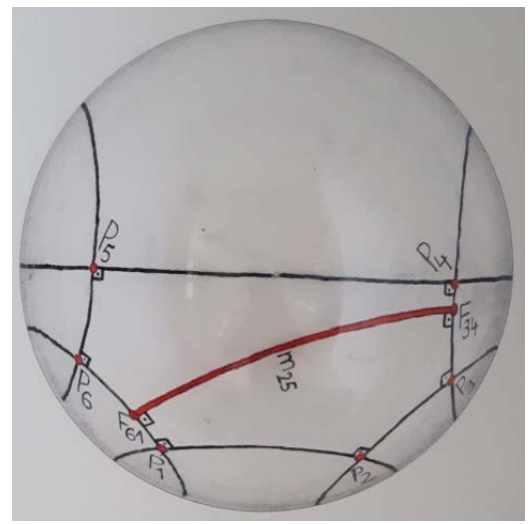

Figure 48. Hyperbolic rectangular hexagon decomposed into two Napier pentagons.

\subsection{Two Triads of Non-Adjacent Sides of the Hyperbolic Rectangular Hexagon as the Counterpart of a Pair of Spherical Polar Triangles}

The sides of the hyperbolic hexagon can be divided into two triads of non-adjacent elements $a=\mathrm{P}_{6} \mathrm{P}_{1}, c=\mathrm{P}_{2} \mathrm{P}_{3}, e=\mathrm{P}_{4} \mathrm{P}_{5}$ and $b=\mathrm{P}_{1} \mathrm{P}_{2}, d=\mathrm{P}_{3} \mathrm{P}_{4}, f=\mathrm{P}_{5} \mathrm{P}_{6}$ (Figure 48).

The two triads ("odd sides" and "even sides") can be viewed the counterpart of a pair of spherical polar triangles (Figure 31), or the rectangular spherical hexagram (Figure 33). From another perspective, they also correspond to the angles and sides of a spherical triangle. The role of spherical angles can be attributed to either of the hyperbolic triads, while the other hyperbolic triad corresponds to the sides of the spherical triangle. Alternatively, the two triads can be taken for the sides of a pair of polar triangles, or for the angles of the same pair of triangles.

\subsection{Noteworthy Lines in the Hyperbolic Rectangular Hexagon}

Following are some examples about the concurrence of some noteworthy lines in the hyperbolic rectangular hexagon, mainly the counterparts of similar theorems on spherical polar triangles. The concurrence can be proved in several ways, for example, by Menelaus or Ceva theorems, or by projecting the hyperbolic figure onto the plane or onto the sphere, prove the assumption in plane or spherical 
geometry, then projecting the result back onto the hyperbolic surface (detailed proofs in [4]).

A pair of polar triangles (Figure 31 and Figure 33) can also be viewed as a rectangular spherical hexagram. It follows that the three common altitudes of the polar triangles are transformed into the common perpendiculars of the opposite sides of the spherical hexagram.

This interpretation can be extended to the hyperbolic rectangular hexagon in Figure 50. On the sphere and on the hyperbolic hemisphere, the three altitudes or common perpendiculars meet in the orthocentre denoted by $\mathrm{H}$ in Figure 49 and Figure 50.

Figure 51 shows the inscribed circle in one triad of sides of the rectangular hyperbolic hexagon. It is tangential to the "odd sides", but not to the "even sides". Figure 52 shows the inscribed circle in the other triad of sides, tangential to the "even sides", but not to the "odd sides". This property corresponds to a similar property of spherical circles inscribed in a pair of polar triangles.

Figure 53 and Figure 54 show the Theorem of Fagnano applied to the hyperbolic rectangular hexagon: The feet of altitudes in a triad of non-adjacent sides are the vertices of an inscribed triangle in which the original altitudes are angle bisectors. The two figures display the inscribed triangles in the "odd triad" and the "even triad, respectively. Again, this property corresponds to the similar statement in a pair of polar triangles on the sphere.

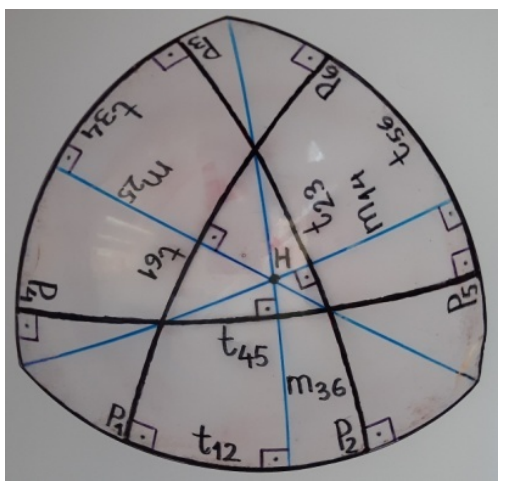

Figure 49. Altitudes of spherical polar triangles (H orthocentre).

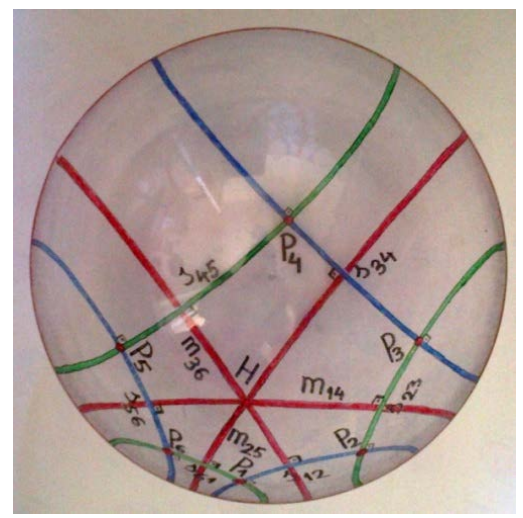

Figure 50. Common perpendiculars of hyperbolic rectangular hexagon ( $\mathrm{H}$ orthocentre). 


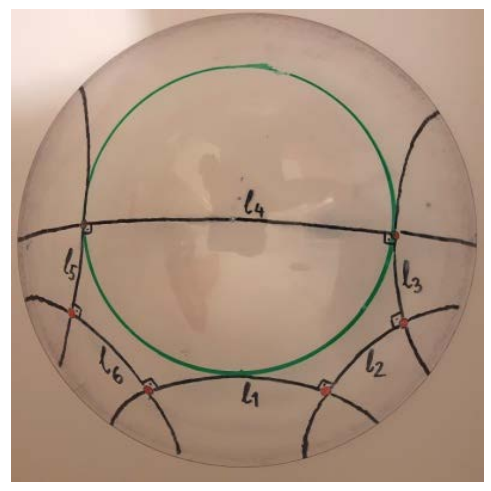

Figure 51. Inscribed circle in the "odd" triad of the hexagon.

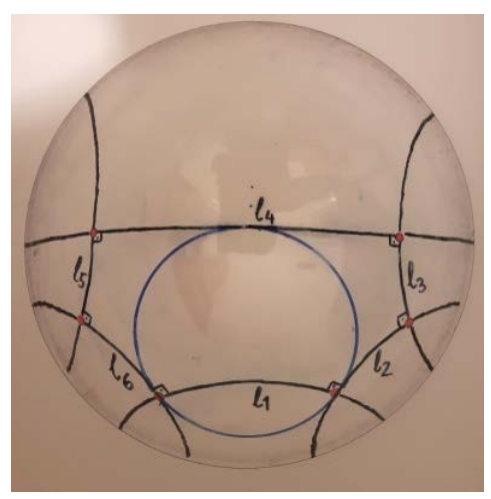

Figure 52. Inscribed circle in the "even" triad of the hexagon.

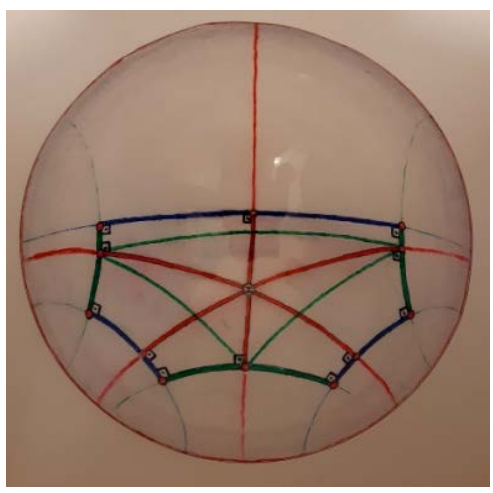

Figure 53. Theorem of Fagnano in one triad of non-adjacent sides.

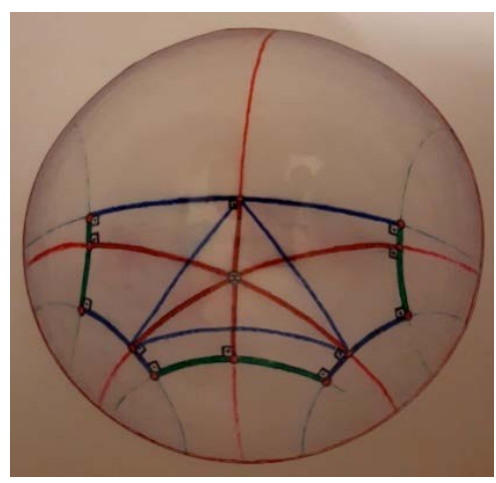

Figure 54. Theorem of Fagnano in the other triad of non-adjacent sides. 


\subsection{The Desargues Configuration on the Hyperbolic Surface}

The Desargues configuration can be displayed in novel forms by the present algebraic approach.

Figure 55 displays the two 6-cycles ("triangles") of the Desargues configuration in the form where all the elements of the 6-cycles are lines, while the "centre" $S$ and "axis" $T$ appear as points.

Figure 56 shows two perspective rectangular hyperbolic hexagons in the form of regular triangles. "Perspective" means in this case that the three products of the corresponding three pairs of sides are concurrent. The "centre" $S$ of perspectivity coincides with the "axis" $T$ of perspectivity, because

$$
a_{1} a_{2}\left|b_{1} b_{2}=a_{3} b_{3}, a_{2} a_{3}\right| b_{2} b_{3}=a_{1} b_{1}, a_{3} a_{1} \mid b_{3} b_{1}=b_{2} a_{2} .
$$

An interesting application of the form of the Desargues theorem in Figure 55 is the Veblen-Young dual theorem as cited by Gévay [21]: If three triangles are perspective from the same point, the three axes of perspectivity of the three pairs of triangles are concurrent. Conversely, if three triangles are perspective from the same line, the three centres of perspectivity of the three pairs of triangles are collinear.

These two theorems can be unified in one theorem in the following way: If the common perpendiculars of the odd sides of three rectangular hexagons are concurrent, the common perpendiculars of the even sides are also concurrent.

This form is more suitable for generalization in higher dimensions than the point-line version.

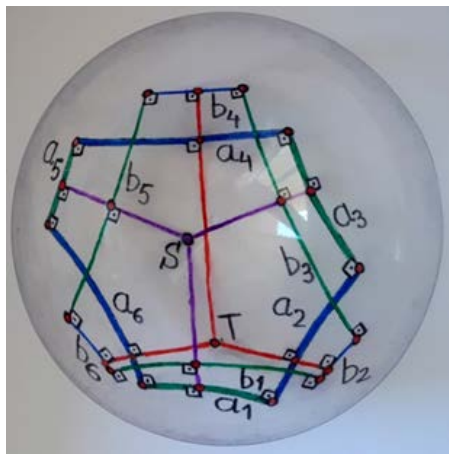

Figure 55. The Desargues configuration: Two perspective rectangular hexagons.

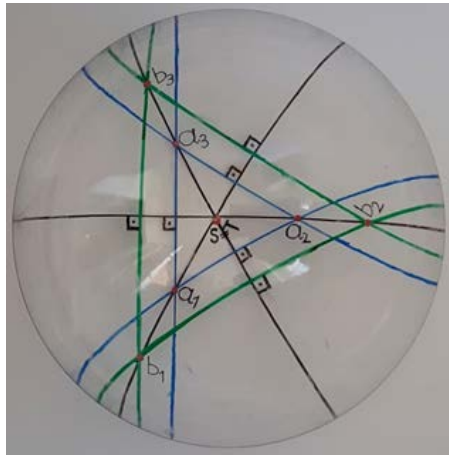

Figure 56. The Desargues configuration: Two regular perspective hyperbolic triangles. 


\section{The Cartesian Coordinate System as a Model of Projective Spheres}

\subsection{Pairs of Numbers-Points and Straight Lines}

As shown in Section One and Section Two, the theory of projective spheres treats points and straight lines as identical elements within a single set, not as dual elements in two separate sets.

In the Cartesian system, a point in the plane is represented by an ordered pair of real numbers $(a, b)$, and a straight line by a linear equation $a x+b=y$ where $a$ and $b$ are constants.

If no distinction is made between points and straight lines, it seems reasonable to say that the ordered pair of real numbers $(a, b)$ represents both the point $(a, b)$ and the straight line $a x+b=y$.

The problem lies in defining the relation of incidence between a point and a straight line. Assume that point $[(+c),(+d)]$ is on straight line $[(+a),(+b)]$, algebraically $(+a)(+c)+(+b)=(+d)$. Conversely, assume that point $[(+a),(+b)]$ is on straight line $[(+c),(+d)]$, that is, $(+c)(+a)+(+d)=(+b)$. From the two equations we get: $(+a)(+c)=(+c)(+a)=(+b)-(+d)=(+d)-(+b)=0$, so $(+b)=(+d)$, and $(+a)(+c)=(+c)(+a)=0$.

The symmetry of the incidence relation works if $(+a)(+c)=(+c)(+a)=0$, but fails in the general case. If point $[(+a),(+b)]$ is on line $[(+c),(+d)]$, then point $[(+c),(+d)]$ is not on line $[(+a),(+b)]$, but on line $[(-a),(+b)]$; that is, if $(+c)(+a)+(+d)=(+b)$, then $(-a)(+c)+(+b)=(+d)$.

There are two ways to escape this trap.

We can accept that the incidence relation is antisymmetric in the above sense: If point $[(+a),(+b)]$ is on line $[(+c),(+d)]$, then point $[(+c),(+d)]$ is on line $[(-a),(+b)]$. This approach is in stark contrast to the geometric perception, which is why I reject it in the present paper (although it certainly has a potential in a different context).

Alternatively, unify two points and two straight lines of the Cartesian system into one basic element whose coordinates are $( \pm a),(+b)$. Our basic element $[( \pm a)$, $(+b)$ ] contains point $(+a),(+b)$, point $(-a),(+b)$, straight line $(+a) x+(+b)=y$ and straight line $(-a) x+(+b)=y$. The sign of a may be either positive or negative, but the sign of $b$ remains the same in each case.

Figure 57 shows a concrete example, the basic element $[( \pm 2),(+5)]$ made of two points and two straight lines reflected to the $y$-axis. Figure 58 illustrates the relation of incidence. The basic elements $[( \pm 2),(+5)][( \pm 4),(-3)]$ are incident to each other. Geometrically, the points of one basic elements are incident to the lines of the other element, and conversely.

\subsection{Classification of Poles and Polars of Elements in a Cartesian Projective Sphere}

In order to create a projective sphere, we have to add ideal elements (ideal points and the ideal line) to the real points and lines of the coordinate system. Follow- 
ing is a list of possible cases with real and ideal elements.

1) Two real points and two real straight lines that are mirror images to the $y$-axis (Figure 57). The point of intersection of the two lines is the midpoint on the $y$-axis between the two points. This is not a reflexive element, because the pole points are not on (not incident to) the polar lines.

2) A real point and a real straight line (Figure 59): The line is the $x$-axis or any other line parallel with it. The pole point is the point of intersection of the line with the $y$-axis. This is a reflexive element with the pole point on the polar line.

3) The ideal point of the $y$-axis and the ideal line: This is also a reflexive element with the pole point on the polar line.

4) The $y$-axis and the ideal point of the $x$-axis. This is not reflexive, because the pole point is not on the polar line.

5) Two straight lines that are mirror images to, and parallel with, the $y$-axis, and the ideal points of two straight lines through the origo (Figure 60). The slope of the two latter straight lines is the same as the distance of the parallel lines from the origo. This element is not reflexive.

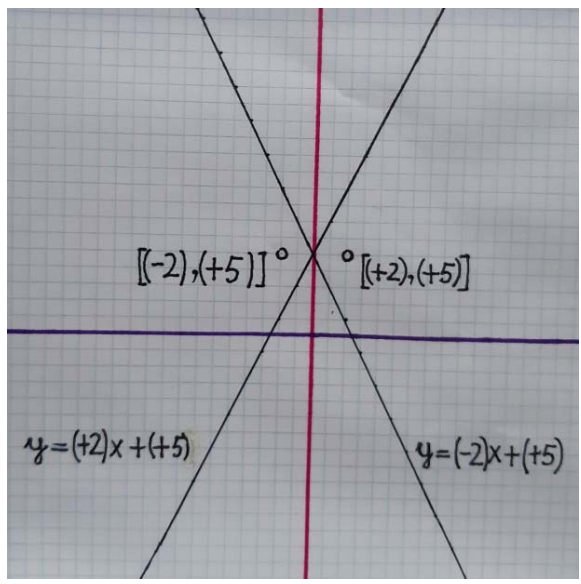

Figure 57. Basic element $[( \pm 2),(+5)]$.

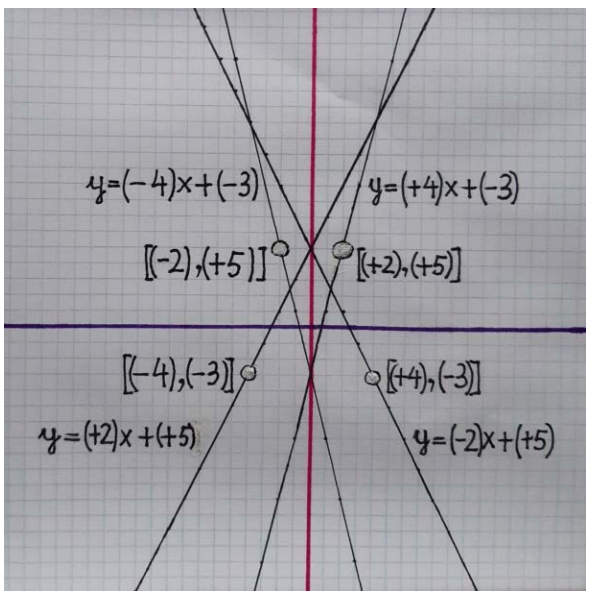

Figure 58. Incident elements $[( \pm 2),(+5)][( \pm 4),(-3)]$. 


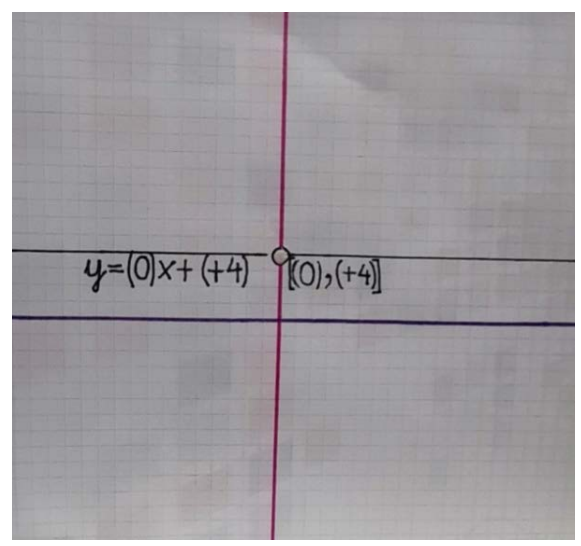

Figure 59. Reflexive element.

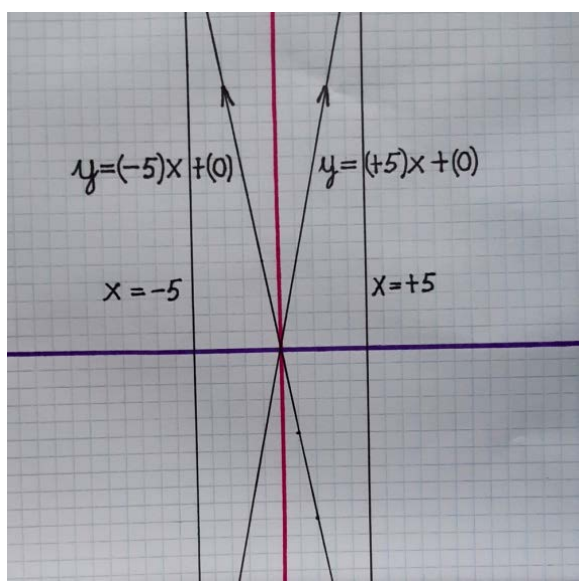

Figure 60. Polar lines parallel with the $y$-axis.

\subsection{Algebraic Condition of the Relation of Incidence}

Two elements $x[( \pm a),(+b)]$ and $y[( \pm c),(+d)]$ are incident if the square of the product of the first coordinates is the same as the square of the difference of the second coordinates: $[( \pm a)( \pm c)]^{2}=[(+d)-(+b)]^{2}$.

\subsection{Algebraic Condition for Reflexive Elements}

If the two elements are the same: $( \pm a)^{\circ}=( \pm c)$, and $(+b)=(+d)$ where the signs of the second coordinates are fixed, element $[( \pm a),(+b)]$ is reflexive if: $[( \pm a)( \pm a)]^{2}=[(+b)-(+b)]^{2}$, so $(+a)=0,(+b)$ is arbitrary. This corresponds to the geometric figure where the polar line of a reflexive element is parallel with the $x$-axis. The pole point is the intersection of the line and the $y$-axis (Figure $59)$.

\subsection{Establishing a Binary Operation in the Cartesian Set}

As shown in Section One, a projective sphere can be defined either by two axioms of a binary relation and/or two axioms of a binary operation (subsection 4, see also [5] [8]).

The two axioms of the relation are: 
Consider a set of elements with a binary relation $R$ defined between any two elements of the set, including the $\boldsymbol{x} \boldsymbol{R} \boldsymbol{x}$ case, for which the following two axioms hold:

$$
\begin{gathered}
x \boldsymbol{R} y=\boldsymbol{x} \boldsymbol{x} \quad \text { (symmetric relation) } \\
\exists ! z \mid x \neq \boldsymbol{y}=\boldsymbol{x} \boldsymbol{R} \mathbf{z} \boldsymbol{y}
\end{gathered}
$$

(any two different elements $\boldsymbol{x} \neq \boldsymbol{y}$ determine one element $\boldsymbol{z}$ for which $\boldsymbol{z} \boldsymbol{R}$ and $\boldsymbol{z} \boldsymbol{R} \boldsymbol{y}$ hold)

The two axioms of the operation are:

$$
\begin{gathered}
x y=y x \quad \text { (commutative property) } \\
(x y)(x z)=x \quad \text { (main axiom) }
\end{gathered}
$$

How can we use the relation of incidence for defining the binary operation in a projective sphere? Given two elements $x[( \pm a),(+b)]$ and $y[( \pm c),(+d)]$; determine an element $z[( \pm e),(+f)]$ which is incident to both $x[( \pm a),(+b)]$ and $y[( \pm c),(+d)]$.

$$
\begin{aligned}
& {[( \pm a)( \pm e)]^{2}=[(+f)-(+b)]^{2}} \\
& {[( \pm c)( \pm e)]^{2}=[(+f)-(+d)]^{2}}
\end{aligned}
$$

From this we get:

$$
( \pm e)=\frac{(+d)-(+b)}{( \pm c)-( \pm a)} ;(+f)=\frac{( \pm c)(+b)-( \pm a)(+d)}{( \pm c)-( \pm a)}
$$

\subsection{An Ambiguity in the Definition}

As shown in the formulas above, two elements are incident to not only one, but two different elements in the general case.

$$
\begin{aligned}
& \left( \pm e_{1}\right)=\frac{(+d)-(+b)}{(+c)-(+a)} ; \\
& \left(+f_{1}\right)=\frac{(+c)(+b)-(+a)(+d)}{(+c)-(+a)} \text { (same for the }(-c),(-a) \text { case) } \\
& \left( \pm e_{2}\right)=\frac{(+d)-(+b)}{(+c)-(-a)} ; \\
& \left(+f_{2}\right)=\frac{(+c)(+b)-(-a)(+d)}{(+c)-(-a)} \quad \text { (same for the }(-c),(+a) \text { case) }
\end{aligned}
$$

I will take this ambiguity into consideration in all further reasoning.

I could not find a suitable principle to exclude one of the two possible outcomes.

\subsection{Proving the Two Axioms of the Operation}

The two axioms are: $x y=y x$ (commutative property), $(x y)(x z)=x$ (main axiom).

1) Commutative property: 
Given two elements $x[(+a),(+b)], \quad y[(+c),(+d)]$, prove that $x y=y x$.

First coordinate (may be positive or negative):

$$
\begin{gathered}
\frac{(+d)-(+b)}{(+c)-(+a)}=\frac{(+b)-(+d)}{(+a)-(+c)} \\
\frac{(+d)-(+b)}{(+c)-(-a)}=\frac{(+d)-(+b)}{(+c)+(+a)}=-\frac{(+b)-(+d)}{(+c)+(+a)}
\end{gathered}
$$

Second coordinate (fixed sign):

$$
\begin{gathered}
\frac{(+c)(+b)-(+a)(+d)}{(+c)-(+a)}=\frac{(+a)(+b)-(+c)(+d)}{(+a)-(+c)} \\
\frac{(+c)(+b)-(-a)(+d)}{(+c)-(-a)}=\frac{(+c)(+b)+(+a)(+d)}{(+c)+(+a)}=\frac{(+a)(+d)+(+c)(+b)}{(+a)+(+c)} \\
=\frac{(-a)(+d)-(+c)(+b)}{(-a)-(+c)}
\end{gathered}
$$

Similar proof for the $x[(+a),(+b)], y[(-c),(+d)]$ case.

2) Main axiom:

Given three elements $x[(+a),(+b)], \quad y[(+c),(+d)], z[(+e)(+f)]$, prove that $(x y)(x z)=x$.

First coordinate (may be positive or negative):

$$
\begin{aligned}
\frac{\frac{e b-a f}{e-a}-\frac{c b-a d}{c-a}}{\frac{f-b}{e-a}-\frac{d-b}{c-a}} & =\frac{(e b-a f)(c-a)-(c b-a d)(e-a)}{(f-b)(c-a)-(d-b)(e-a)} \\
& =\frac{(-e b-c f+a f+d e-a b+c d) a}{+b e+c f-a f-d e+a d-b c}=-a
\end{aligned}
$$

Second coordinate (fixed sign):

$$
\begin{aligned}
& \frac{\frac{(f-b)}{e-a} \frac{(c b-a d)}{c-a}-\frac{(e b-a f)}{e-a} \frac{(d-b)}{c-a}}{\frac{f-b}{e-a}-\frac{d-b}{c-a}} \\
& =\frac{(f-b)(c-a)(c b-a d)(e-a)-(d-b)(c-a)(e b-a f)(e-a)}{(f-b)(c-a)-(d-b)(e-a)} \\
& =\frac{(+f c-c b-d e+e b-a d-a f) b}{+f c-b c-e d+e b-a d-a f}=+b
\end{aligned}
$$

Similar proofs for other combinations of $( \pm a),( \pm c),( \pm e)$. Q.E.D.

\subsection{Klein Spheres (K-Spheres)}

As shown in Section One, the Klein sphere or K-sphere is a closed set in the theory of projective spheres (subsection 7, Model 1). It consists of three different elements, each of which is incident to the other two. It follows from the definition that reflexive elements cannot occur in a $\mathrm{K}$-sphere.

The condition of incidence in the $\mathrm{K}$-sphere in the Cartesian system: 
Given three elements $x[( \pm a),(+b)], y[( \pm c),(+d)], z[( \pm e)(+f)]$

$$
\begin{aligned}
& {[( \pm a)( \pm c)]^{2}=[(+d)-(+b)]^{2}} \\
& {[( \pm c)( \pm e)]^{2}=[(+f)-(+d)]^{2}} \\
& {[( \pm e)( \pm a)]^{2}=[(+b)-(+f)]^{2}}
\end{aligned}
$$

Example (Figure 61):

Elements $x[( \pm 2),(+4)], y[( \pm 6),(-8)], z[( \pm 3)(+10)]$ make a K-sphere:

$x[( \pm 2),(+4)], y[( \pm 6),(-8)]$ are incident, because $(+2)(+6)=(+4)-(-8)$.

$y[( \pm 6),(-8)], z[( \pm 3)(+10)]$ are incident, because

$(+6)(+3)=(+10)-(-8)$.

$z[( \pm 3),(+10)], x[( \pm 2),(+4)]$ are incident, because

$(+3)(+2)=(+10)-(+4)$.

In the K-sphere, the product of any two different elements is the third element. The product of any two adjacent elements in Table 11 is the next element below (If point $s[(+a),(+b)]$ is incident to line $t[(+c),(+d)]$, then line $s[(+a),(+b)]$ is incident to line $t[(-c),(+d)])$.

The order is important because of the ambiguity of the operation. If $x y=z$ is given in the form $x[(+3),(+10)] y[(+2),(+4)]=z[(+6),(-8)]$, the product of row 1 and row 2 gives row 3 , then $z x=y$ is given by $z[(+6),(-8)] x[(-3),(+10)]=y[(-2),(+4)]$, the product of row 3 and row 4 gives row 5 .

\subsection{Hesse Theorem in the Cartesian Projective Sphere}

\section{Theorem 15:}

The following equations hold for elements $x(a, b), y(c, d), z(e, f)$ :

$$
x y z|y z x=y z x| z x y=z x y \mid x y z .
$$

Proof:

The three associative elements can be developed in the following form:

$$
\begin{aligned}
& \underline{\mathbf{x y z}}=\frac{+b c-a d-c f+a f}{+d-b-c e+a e}, \frac{+d f-b f-b c e+a d e}{+d-b-c e+a e} \\
& \underline{\mathbf{y z} x}=\frac{+d e-c f-b e+b c}{+f-d-e a+a c}, \frac{+b f-b d-a d e+a c f}{+f-d-e a+a c} \\
& \underline{\mathbf{z x y}}=\frac{+a f-b e-a d+e d}{+b-f-a c+e c}, \frac{+b d-d f-a c f+b c e}{+b-f-a c+e c}
\end{aligned}
$$

If we perform the operations $x y z|y z x=y z x| z x y=z x y \mid x y z$ with these expressions, we get 16 addends in each expression which prove the theorem. The roles of the coordinates in the above formulas are symmetrical. The detailed calculation is simple, but lengthy and tedious, so I skip it.

This result supports the hypothesis that in some cases it is worthwhile to study projective spheres with the Hesse configuration as a third axiom, in addition to the two basic axioms $a b=b a$ and $a b \mid a c=a$. 


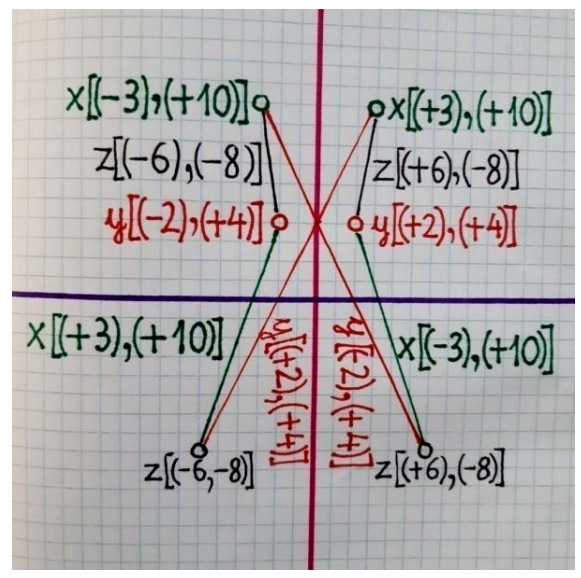

Figure 61. Klein sphere in the Cartesian system.

Table 11. Construction of a K-sphere.

\begin{tabular}{ccc}
\hline. & $x[(+3),(+10)]$ \\
2. & $y[(+2),(+4)]$ \\
3. & $x[(+6),(-8)]$ \\
4. & $y[(-2),(+10)]$ \\
5. & $z[(-6),(-8)]$ \\
6. & $x[(+3),(+10)]$ \\
1. & $y[(+2),(+4)]$ \\
2. & $x[(+3),(+10)]$ \\
3. & $y[(+2),(+4)]$ \\
4. & $z[(+6),(-8)]$ \\
5. & $x[(-3),(+10)]$ \\
6. & $\ldots$ \\
\hline
\end{tabular}

\subsection{The $a$ a Product}

As described in Theorem 1, the product aa cannot be adequately defined for all elements of the set. How is this property manifested in the Cartesian sphere?

The operation in the form $x[(+a),(+b)] x[(+a),(+b)]$ cannot be uniquely determined, because it gives $x[(+a),(+b)] x[(+a),(+b)]=x x\left[\frac{0}{0}, \frac{0}{0}\right]$. In contrast, the other form $x[(+a),(+b)] x[(-a),(+b)]$ gives the reasonable product $x x\left[\frac{0}{2 a}, \frac{2 a b}{2 a}\right]=[0, b]$. However, the $x x$ operation with any reflexive element $x[(0),(+b)] x[(0),(+b)]=x x\left[\frac{0}{0}, \frac{0}{0}\right]$ cannot be interpreted by this definition.

Therefore, the original assumption remains valid in the Cartesian projective sphere: 
The expression $x x$ cannot be uniquely determined for all elements in the set with at least two different elements.

\subsection{Differentiable Functions}

When a point in the plane approaches another point, the two points define a unique straight line through them. If the two points coincide, an infinite number of straight lines can be drawn through the point. So there is no unique result of the $X X$ operation.

However, a differentiable function has a precisely defined tangent line in each of its points. The tangent is the limit of an infinite series of secants each of which passes through the given point and another point of the function. By the operation as defined in the projective sphere, this means that if $x$ is an element of the differentiable function, operation $x x$ is uniquely defined by the tangent at the given point of the function. The classical calculus determines the slope of the tangent as the limit of division with an arbitrarily small number $\Delta x$. The algebra of projective spheres defines the tangent as the product of an element of a differentiable function multiplied by itself.

How can we determine the coordinates of the tangent? By definition, the tangent is incident to the point. There is no difference between points and lines in a projective sphere, so the condition of incidence can be applied to determine the coordinates of the tangent.

Before giving the general formula, we begin with concrete examples:

1) Given a point of the function $y=x^{2}$ : first coordinate $x$, second coordinate $x^{2}$. We want to determine the second coordinate of the tangent in this point.

Figure 62 shows the polar lines of the tangent elements. Figure 63 shows the pole points of the same tangent elements.

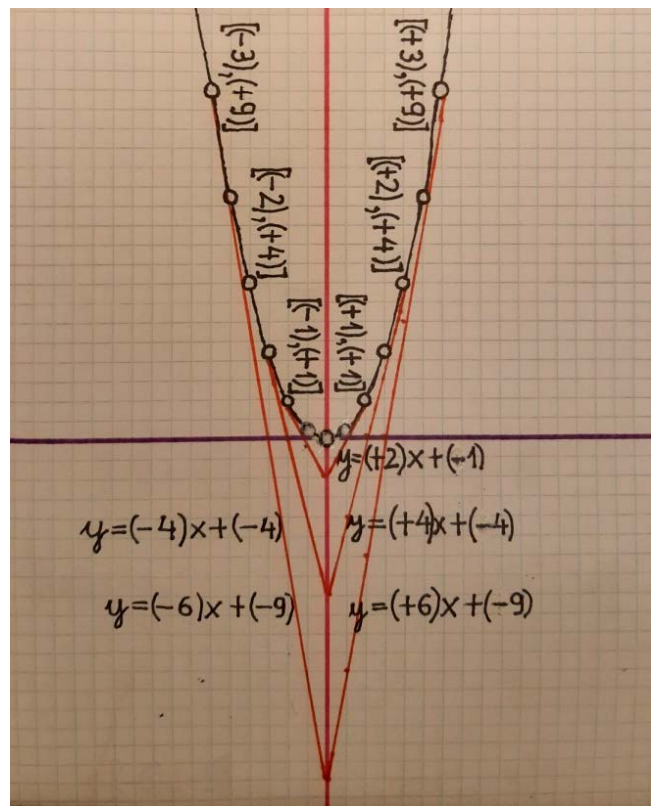

Figure 62. Tangents as lines of the $y=x^{2}$ function. 


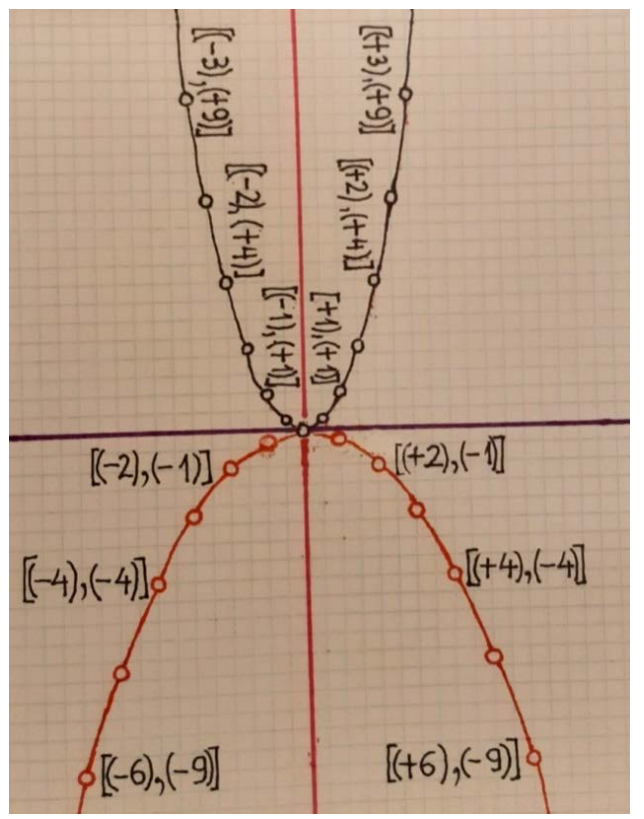

Figure 63. Tangents as points of the $y=x^{2}$ function.

The first coordinate of the point is $x$, the second coordinate is $x^{2}$. If we know that the first coordinate of the tangent is $y^{\prime}=2 x$, what is the second coordinate?

The tangent is incident to the point, so we can apply the condition of incidence: The product of the first coordinates is equal to the difference of the second coordinates. The product of the first coordinates in this case is $2 x^{2}$. The second coordinate of the tangent is either $-x^{2}$ or $3 x^{2}$, but the geometric image of the function shows that the only possible solution is $-x^{2}$.

Furthermore, we can construct the normal to the parabola which is the product of the point and the tangent in the projective sphere. The three elements form a K-sphere at almost every point of the differentiable function. Table 12 shows the coordinates of these elements displayed as points.

Exceptions are the points in which the tangents are parallel with the $x$-axis. hese points are reflective elements of the perspective sphere representing local extrema (maximum or minimum points), or certain inflection points of the function (Figure 64).

Figure 65 shows two K-spheres assigned to two elements of the original $y=x^{2}$ parabola, as concrete numerical examples: $[( \pm 1)(+1)],[( \pm 2)(-1)][( \pm 2)(+3)]$, and $[( \pm 2)(+4)][( \pm 4)(-4)][( \pm 4)(+12)]$. Table 13 shows the respective coordinates.

1) Another example is the unit circle in Figure 66. The red curves show the pole points of the tangent function.

2) The hyperbola in Figure 67 is another example. The third row of Table 14 shows the coordinates of the normal, that is, the product of the point and the tangent, thus establishing K-spheres to every point. Figure 67 only displays the hyperbola and the tangent function. 


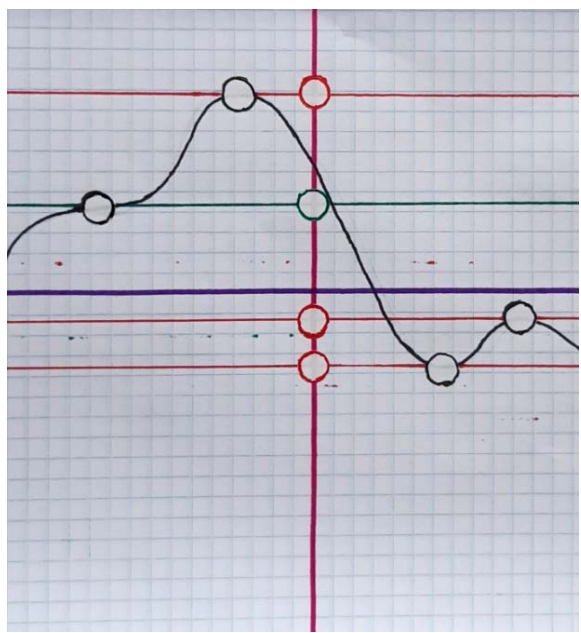

Figure 64. Tangents of a function parallel with the $x$-axis.

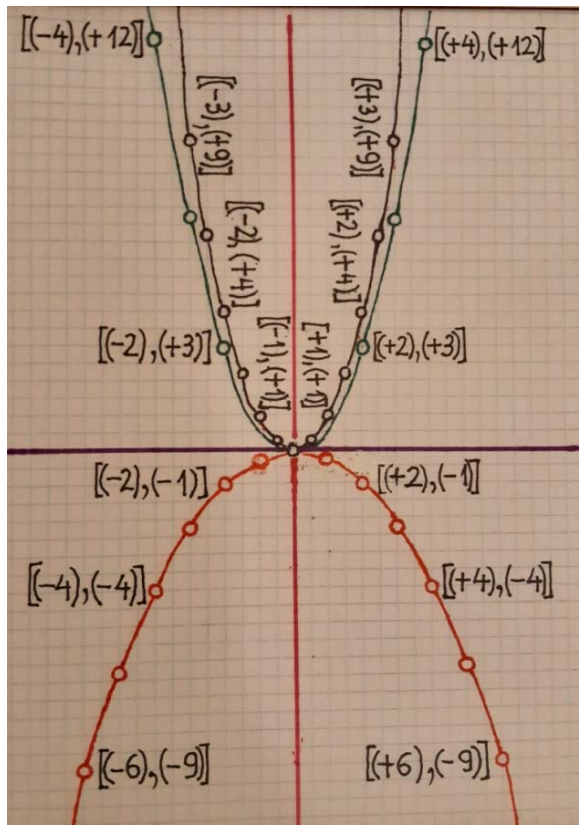

Figure 65. The parabola function, its tangents and normals, all displayed as points.

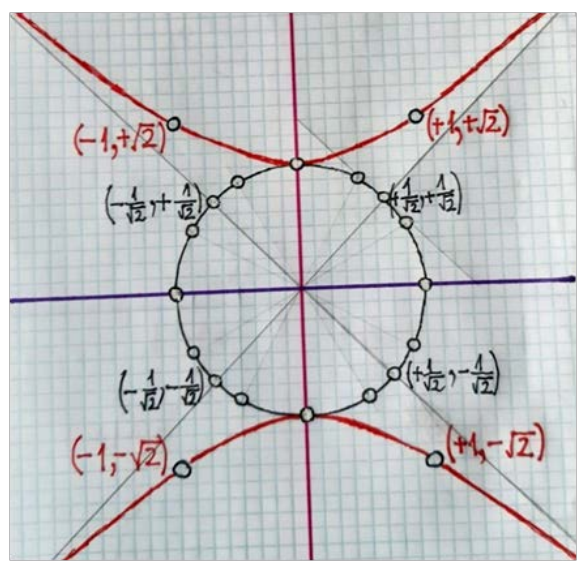

Figure 66. The unit circle. 


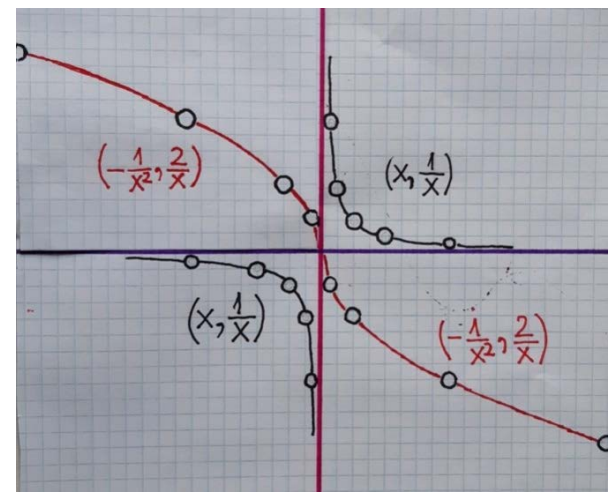

Figure 67. The hyperbola.

Table 12. Coordinates of the parabola, its tangents and normals.

$\begin{array}{ccc} \pm x & x^{2} & \text { points of the parabola } \\ \pm 2 x & -x^{2} & \text { tangents } \\ \pm 2 x & 3 x^{2} & \text { normals (products of points and tangents) }\end{array}$

Table 13. Coordinates of the unit circle and its tangents.

\begin{tabular}{ccc}
\hline $\pm x$ & $-\sqrt{1-x^{2}}$ & (14) \\
$\pm \frac{x}{\sqrt{1-x^{2}}}$ & $-\frac{1}{\sqrt{1-x^{2}}}$ \\
\hline
\end{tabular}

Table 14. Coordinates of the hyperbola, its tangents and normals.

$\begin{array}{cc} \pm x & \frac{1}{x} \\ \pm \frac{1}{x^{2}} & \frac{2}{x} \\ \pm \frac{x}{x^{3}+1} & \frac{2 x^{3}+1}{\left(x^{3}+1\right) x}\end{array}$

\section{Closing Remarks}

I am far from assigning any kind of exclusivity or superiority to the present algebraic-axiomatic system compared to other systems. I am just saying that this is a meaningful construction among many other possible options. It is up to the user to decide which system is more suitable for solving a mathematical or educational problem.

Certainly, this work poses many unresolved issues and incomplete analogies. After five decades of research, however, it is high time that I summarized the results so far in the hope that future researchers will be able to use and improve what I have done.

I have been immersed in the theory of projective spheres for many years because they have given me joy and satisfaction. I ask future teachers of this topic to share this joy, satisfaction and self-confidence with their students. 


\section{Conflicts of Interest}

The author declares no conflicts of interest regarding the publication of this paper.

\section{References}

[1] Baer, R. (1946) Polarities in Finite Projective Planes. Bulletin of the American Mathematical Society, 52, 77-93. https://doi.org/10.1090/S0002-9904-1946-08506-7

[2] Gunn, C.G. (2014) Introduction to Projective Geometric Algebra. https://wordpress.discretization.de/vismathws12/2014/02/28/introduction-to-geom etric-algebra-iii

[3] Leibniz, G.W. (1679) Letter to Christian Huygens. https://link.springer.com/chapter/10.1007/978-94-010-1426-7_28

[4] Lénárt, I. (2020) Projective Spheres in Three Geometries. ResearchGate.

[5] Devidé, V. (1965) Plane Pre-Projective Geometries. Period. Math., 20, 251-260.

[6] Devidé, V. (1969) Some Properties of Pre-Geometric Binary Relations. Publ. Elektrotehn. Fak. u Beogradu, No. 301, 61-78.

[7] Kárteszi, F. and Lénárt, I. (1983) A Generalization of the Concept of Projective Plane. Rendiconti di Matematica, 2, 643-663. (In Italian)

[8] Coxeter, H.S.M. (1974) Projective Geometry. 2nd Edition, Toronto Univ. Press, Toronto, Ch. 10. https://doi.org/10.1007/978-1-4612-6385-2

[9] Kárteszi, F. (1972) Introduction to Finite Geometries. Akadémiai Kiadó, Budapest.

[10] Gleason, A.M. (1956) Finite Fano Planes. American Journal of Mathematics, 78, 797-807. https://doi.org/10.2307/2372469

[11] Struik, D. (1948) A Concise History of Mathematics. Dover, New York. https://doi.org/10.1038/scientificamerican1248-44

[12] Hessenberg, G. (1905) Beweis des Desargues'sche Satzes aus dem Pascalschen. Mathematische Annalen, 6, 161-172. https://doi.org/10.1007/BF01457558

[13] Dénes, J. and Keedwell, A.D. (1974) Latin Squares and Their Applications. Akadémiai Kiadó, Budapest, 58-60.

[14] Devidé, V. (1969) A Proof of Two Theorems of R. Baer. Glasn. Mat. Tom 4(24), No. 2, 255-257.

[15] Veblen, O. and Wedderburn, J.H.M. (1907) Non-Desarguesian and Non-Pascalian Geometries. Transactions of the American Mathematical Society, 8, 379-388. https://doi.org/10.1090/S0002-9947-1907-1500792-1

[16] Lénárt, I. (1978) Irreguláris polaritás véges síkon (Irregular Polarity in Finite Planes). Matematikai Lapok, János Bolyai Mathematical Society, 31, 135-141.

[17] Szilassi, L. (2019) Personal Communication.

[18] Morley, F. (1897) On a Regular Rectangular Configuration of Ten Lines. Proceedings of the London Mathematical Society, 29, 670-673. https://doi.org/10.1112/plms/s1-29.1.670

[19] Gauss, C.F. (1866) Pentagramma Mirificum. Werke, Band III: Analysis. Königliche Gesellschaft der Wissenschaften, Göttingen, 481-490.

[20] Coxeter, H.S.M. (1971) Frieze Patterns. Acta Arithmetica, 18, 297-310. http://matwbn.icm.edu.pl/ksiazki/aa/aa18/aa18132.pdf https://doi.org/10.4064/aa-18-1-297-310 
[21] Gévay, G. (2018) Pascal's Triangle of Configurations. In: Conder, M.D.E., Deza, A. and Weiss, A.I., Eds., Discrete Geometry and Symmetry, Springer, Berlin, 181-200. https://doi.org/10.1007/978-3-319-78434-2_10 